\title{
Chemistry and Biology of Diazonamide A: First Total Synthesis and Confirmation of the True Structure
}

K. C. Nicolaou,* David Y.-K. Chen, Xianhai Huang, Taotao Ling, Marco Bella, and Scott A. Snyder

Department of Chemistry and The Skaggs Institute for Chemical Biology

The Scripps Research Institute, 10550 North Torrey Pines Road, La Jolla, California 92037 and Department of Chemistry and Biochemistry, University of California, San Diego, 9500 Gilman Drive, La Jolla, California 92093

Corresponding Author: kcn@scripps.edu

\section{Supporting Information}

\section{Experimental Data for Compounds}

General Procedures. All reactions were carried out under an argon atmosphere with dry solvents under anhydrous conditions, unless otherwise noted. Dry tetrahydrofuran (THF), toluene, diethyl ether $\left(\mathrm{Et}_{2} \mathrm{O}\right)$ and methylene chloride $\left(\mathrm{CH}_{2} \mathrm{Cl}_{2}\right)$ were obtained by passing commercially available pre-dried, oxygen-free formulations through activated alumina columns.

Yields refer to chromatographically and spectroscopically $\left({ }^{1} \mathrm{H}\right.$ NMR) homogeneous materials, unless otherwise stated. Reagents were purchased at the highest commercial quality and used without further purification, unless otherwise stated. Reactions were monitored by thin-layer chromatography (TLC) carried out on $0.25 \mathrm{~mm}$ E. Merck silica gel plates (60F-254) using UV light as visualizing agent and an ethanolic solution of phosphomolybdic acid and cerium sulfate, and heat as developing agents. E. Merck silica gel (60, particle size $0.040-0.063 \mathrm{~mm})$ was used for flash column chromatography. Preparative thin-layer chromatography (PTLC) separations were carried out on 0.25 or $0.50 \mathrm{~mm}$ E. Merck silica gel plates (60F-254). NMR spectra were recorded on Bruker DRX-600, DRX-500, AMX-500 or AMX-400 instruments and calibrated using residual undeuterated solvent as an internal reference. The following abbreviations were used to explain the multiplicities: $\mathrm{s}=$ singlet, $\mathrm{d}=$ doublet, $\mathrm{t}=$ triplet, $\mathrm{q}=$ quartet, $\mathrm{m}=$ multiplet, 
quin $=$ quintuplet, sext $=$ sextet, sep $=$ septet, br $=$ broad, app $=$ apparent, $A B=A B$ quartet. IR spectra were recorded on a Perkin-Elmer 1600 series FT-IR spectrometer. Electrospray ionization (ESI) mass spectrometry (MS) experiments were performed on an API 100 Perkin Elmer SCIEX single quadrupole mass spectrometer at $4000 \mathrm{~V}$ emitter voltage. High-resolution mass spectra (HRMS) were recorded on a VG ZAB-ZSE mass spectrometer using MALDI (matrix-assisted laser-desorption ionization).

7-Bromoisatin (21). Sodium sulfate (25.56 g, $180 \mathrm{mmol}, 18$ equiv), a solution of 2bromoaniline (1.72 g, $10.0 \mathrm{mmol}, 1.0$ equiv) in water $(36 \mathrm{~mL})$, concentrated $\mathrm{HCl}(1.35 \mathrm{~mL}, 30.0$ mmol, 3.0 equiv), and a solution of $\mathrm{HONH}_{2} \bullet \mathrm{HCl}(2.1 \mathrm{~g}, 30.0 \mathrm{mmol}, 3.0$ equiv) in water $(10 \mathrm{~mL})$ were added sequentially to a solution of chloral hydrate $(1.81 \mathrm{~g}, 11.0 \mathrm{mmol}, 1.1$ equiv) in water $(24 \mathrm{~mL})$ at $25^{\circ} \mathrm{C}$. The resulting suspension was then warmed to $80{ }^{\circ} \mathrm{C}$ and stirred for 1 hour. Upon completion, the reaction flask contents were cooled to $0{ }^{\circ} \mathrm{C}$, yielding 2bromohydroxyiminoacetanilide as a yellow solid (1.75 g, 72\% yield) which was collected by filtration. Next, this intermediate $(1.75 \mathrm{~g}, 7.20 \mathrm{mmol}, 1.0$ equiv) was added to a flask containing hot $\left(50{ }^{\circ} \mathrm{C}\right)$ concentrated $\mathrm{H}_{2} \mathrm{SO}_{4}(15 \mathrm{~mL})$ at a rate such that the internal temperature of the solution did not rise above $65^{\circ} \mathrm{C}$. Upon completion, the reaction flask contents were then heated to $80{ }^{\circ} \mathrm{C}$ and stirred for an additional 15 minutes. The reaction mixture was then cooled to 25 ${ }^{\circ} \mathrm{C}$, poured into ice water, and extracted with EtOAc $(3 \times 25 \mathrm{~mL})$. The combined organic layers were then washed with water $(50 \mathrm{~mL})$ and brine $(50 \mathrm{~mL})$, dried $\left(\mathrm{MgSO}_{4}\right)$, and concentrated. The resultant red residue was purified by flash column chromatography (silica gel, EtOAc/hexanes, 1:1) to give 7-bromoisatin $\left(0.781 \mathrm{~g}, 48 \%\right.$ yield) as a red amorphous solid. 21: $\mathrm{R}_{f}=0.48$ (silica gel, EtOAc/hexanes, 1:1); IR (film) $v_{\max } 3097,1736,1610,1468,1425,1315,1167,1124,956$, $757 \mathrm{~cm}^{-1} ;{ }^{1} \mathrm{H} \mathrm{NMR}\left(600 \mathrm{MHz}, \mathrm{CDCl}_{3}\right) \delta 8.24$ (br s, $\left.1 \mathrm{H}\right), 7.70(\mathrm{~d}, J=7.9 \mathrm{~Hz}, 1 \mathrm{H}), 7.57(\mathrm{~d}, J=$ $7.5 \mathrm{~Hz}, 1 \mathrm{H}), 7.05(\mathrm{t}, J=7.9 \mathrm{~Hz}, 1 \mathrm{H}) ;{ }^{13} \mathrm{C} \mathrm{NMR}\left(150 \mathrm{MHz}, \mathrm{CDCl}_{3}\right) \delta 182.4,158.4,148.0$, 140.8, 125.0, 124.4, 119.5; HRMS (MALDI-FTMS) calcd for $\mathrm{C}_{8} \mathrm{H}_{4} \mathrm{BrNO}_{2} \mathrm{Na}^{+}\left[\mathrm{M}+\mathrm{Na}^{+}\right]$ 247.9318, found 247.9321 . 
Tertiary alcohol 22. Phenylmagnesium chloride $(0.58 \mathrm{~mL}, 1.9 \mathrm{M}$ in THF, $1.10 \mathrm{mmol}$, 2.5 equiv) was added dropwise to a solution of 7-bromoisatin ( $0.100 \mathrm{~g}, 0.442 \mathrm{mmol}, 1.0$ equiv) in THF $(2.0 \mathrm{~mL})$ at $0{ }^{\circ} \mathrm{C}$. After stirring for 5 minutes at $0{ }^{\circ} \mathrm{C}$, the reaction mixture was quenched by the addition of saturated aqueous $\mathrm{NH}_{4} \mathrm{Cl}(25 \mathrm{~mL})$, poured into water $(50 \mathrm{~mL})$, and extracted with EtOAc $(3 \times 25 \mathrm{~mL})$. The combined organic layers were then washed with water $(50 \mathrm{~mL})$ and brine $(50 \mathrm{~mL})$, dried $\left(\mathrm{MgSO}_{4}\right)$, and concentrated. The resultant yellow residue was purified by flash column chromatography (silica gel, EtOAc/hexanes, 1:1) to give tertiary alcohol 22 (0.129 g, 96\% yield) as a light yellow oil. 22: $\mathrm{R}_{f}=0.40$ (silica gel, EtOAc/hexanes, $\left.1: 1\right) ;{ }^{1} \mathrm{H}$ NMR (400 MHz, $\left.\mathrm{CDCl}_{3}\right) \delta 8.27(\mathrm{~s}, 1 \mathrm{H}), 7.41-7.35(\mathrm{~m}, 3 \mathrm{H}), 7.33-7.28(\mathrm{~m}, 3 \mathrm{H}), 7.17(\mathrm{~d}, J=$ $7.3 \mathrm{~Hz}, 1 \mathrm{H}), 6.93(\mathrm{dd}, J=8.2,7.6 \mathrm{~Hz}, 1 \mathrm{H}), 4.03($ br s, $1 \mathrm{H}) ;{ }^{13} \mathrm{C} \mathrm{NMR}\left(150 \mathrm{MHz}, \mathrm{CDCl}_{3}\right) \delta$ $178.3,139.8,139.3,133.3,128.7,128.6,125.2,124.8,124.1,103.4,28.3$; HRMS (MALDIFTMS) calcd for $\mathrm{C}_{14} \mathrm{H}_{10} \mathrm{BrNO}_{2} \mathrm{Na}^{+}\left[\mathrm{M}+\mathrm{Na}^{+}\right]$325.9787, found 325.9792 .

Phenolic lactam 24. A solution of $\mathrm{TfOH}\left(0.81 \mathrm{~mL}, 8.55 \mathrm{mmol}, 5.0\right.$ equiv) in $\mathrm{CH}_{2} \mathrm{Cl}_{2}$ $(1.0 \mathrm{~mL})$ was added dropwise to a solution of tertiary alcohol $23(0.52 \mathrm{~g}, 1.71 \mathrm{mmol}, 1.0$ equiv) and acetylated tyrosine methyl ester $23(0.59 \mathrm{~g}, 1.80 \mathrm{mmol}, 1.05$ equiv $)$ in $\mathrm{CH}_{2} \mathrm{Cl}_{2}(10 \mathrm{~mL})$ at 0 ${ }^{\circ} \mathrm{C}$. After 20 minutes of stirring at $0^{\circ} \mathrm{C}$, the reaction mixture was poured carefully into a cold $(0$ $\left.{ }^{\circ} \mathrm{C}\right)$ solution of saturated aqueous $\mathrm{NaHCO}_{3}(50 \mathrm{~mL})$, ensuring that the aqueous layer of the resultant biphasic system was slightly basic as measured by standard $\mathrm{pH}$ paper. This mixture was then poured into water $(50 \mathrm{~mL})$ and extracted with EtOAc $(3 \times 50 \mathrm{~mL})$. The combined organic layers were washed with water $(50 \mathrm{~mL})$ and brine $(50 \mathrm{~mL})$, dried $\left(\mathrm{MgSO}_{4}\right)$, and concentrated to give the desired intermediate free amine $(0.81 \mathrm{~g}, 98 \%$ yield $)$ as a white foam. Carrying this material forward without any further purification, this intermediate product $(0.81 \mathrm{~g}$, $1.67 \mathrm{mmol}, 1.0$ equiv) was dissolved in $\mathrm{CH}_{2} \mathrm{Cl}_{2}(5 \mathrm{~mL})$, and then $\mathrm{Ac}_{2} \mathrm{O}(0.81 \mathrm{~mL}, 8.55 \mathrm{mmol}, 5.0$ equiv) was added at $25^{\circ} \mathrm{C}$. After 20 minutes of stirring at $25^{\circ} \mathrm{C}$, the reaction mixture was concentrated directly, and the resultant yellow residue was purified by flash column chromatography (silica gel, EtOAc/hexanes, 2:1) to give phenolic lactam 24 (0.78 g, 84\% yield over two steps) as a clear oil. 24: $\mathrm{R}_{f}=0.17$ (silica gel, EtOAc/hexanes, 3:1); IR (film) $v_{\max } 3272$, 
1719, 1649, 1614, 1508, 1443, 1367, 1214, 1126, 1032, 908, $732 \mathrm{~cm}^{-1} ;{ }^{1} \mathrm{H}$ NMR (600 MHz, $\mathrm{CDCl}_{3}, 1: 1$ mixture of diastereomers $) \delta 9.54(\mathrm{~s}, 1 \mathrm{H}), 9.50(\mathrm{~s}, 1 \mathrm{H}), 8.99(\mathrm{~s}, 1 \mathrm{H}), 8.92(\mathrm{~s}, 1 \mathrm{H})$, $7.34(\mathrm{~d}, J=7.9 \mathrm{~Hz}, 1 \mathrm{H}), 7.33(\mathrm{~d}, J=7.9 \mathrm{~Hz}, 1 \mathrm{H}), 7.27-7.19(\mathrm{~m}, 5 \mathrm{H}), 6.99(\mathrm{t}, J=7.4 \mathrm{~Hz}, 1 \mathrm{H})$, 6.93-6.88 (m, $2 \mathrm{H}), 6.79(\mathrm{~d}, J=5.7 \mathrm{~Hz}, 1 \mathrm{H}), 6.40(\mathrm{~d}, J=7.9 \mathrm{~Hz}, 1 \mathrm{H}), 6.38(\mathrm{~d}, J=7.9 \mathrm{~Hz}, 1 \mathrm{H})$, $4.77(\mathrm{dd}, J=13.1,5.7 \mathrm{~Hz}, 1 \mathrm{H}), 4.73$ (dd, $J=13.6,6.1 \mathrm{~Hz}, 1 \mathrm{H}), 3.60$ (s, $3 \mathrm{H}), 3.47$ (s, $3 \mathrm{H}), 2.98$ $(\mathrm{dd}, J=14.0,5.7 \mathrm{~Hz}, 1 \mathrm{H}), 2.96(\mathrm{dd}, J=12.3,3.5 \mathrm{~Hz}, 1 \mathrm{H}), 2.89$ (dd, $J=13.1,5.7 \mathrm{~Hz}, 1 \mathrm{H}), 2.87$ $(\mathrm{dd}, J=13.6,5.3 \mathrm{~Hz}, 1 \mathrm{H}), 1.83(\mathrm{~s}, 3 \mathrm{H}), 1.74(\mathrm{~s}, 3 \mathrm{H}) ;{ }^{13} \mathrm{C} \mathrm{NMR}\left(150 \mathrm{MHz}, \mathrm{CDCl}_{3}, 1: 1\right.$ mixture of diastereomers) $\delta 182.0,181.9,172.3,170.5,170.4,155.2$ (2 C), 140.1, 139.8, 138.9, 134.0, 131.3, 131.2, 130.8 (2 C), 130.2, 130.1, 128.9, 128.8, 128.0, 127.9, 127.5, 127.3, 127.0 (2 C), $126.5,126.1,125.6,124.2$ (2 C), 119.0, 118.6, 103.7, 103.6, 63.3, 63.0, 60.5, 53.3, 52.5, 52.2, 37.1, 37.0, 22.8, 22.6; HRMS (MALDI-FTMS) calcd for $\mathrm{C}_{26} \mathrm{H}_{23} \mathrm{BrN}_{2} \mathrm{O}_{5} \mathrm{H}^{+}\left[\mathrm{M}+\mathrm{H}^{+}\right]$523.0863, found 523.0872 .

Aminal 27. $\mathrm{BH}_{3} \cdot \mathrm{SMe}_{2}(0.023 \mathrm{~mL}, 0.383 \mathrm{mmol}, 4.0$ equiv $)$ was added to a solution of lactam $24\left(0.050 \mathrm{~g}, 0.0957 \mathrm{mmol}, 1.0\right.$ equiv) in THF $(1.5 \mathrm{~mL})$ at $0{ }^{\circ} \mathrm{C}$. After 10 minutes of stirring at $0{ }^{\circ} \mathrm{C}$, the reaction mixture was warmed slowly to $25^{\circ} \mathrm{C}$ and stirred for 2 additional hours. Upon completion, the reaction mixture was quenched by the addition of saturated aqueous $\mathrm{NaHCO}_{3}(10 \mathrm{~mL})$ and extracted with EtOAc $(3 \times 15 \mathrm{~mL})$. The combined organic layers were then washed with water $(50 \mathrm{~mL})$ and brine $(50 \mathrm{~mL})$, dried $\left(\mathrm{MgSO}_{4}\right)$, and concentrated. The resultant light yellow residue was purified by flash column chromatography (silica gel, EtOAc/hexanes, 3:1) to give aminal $27\left(0.019 \mathrm{~g}, 40 \%\right.$ yield) as a clear oil. 27: $\mathrm{R}_{f}=0.33$ (silica gel, EtOAc/hexanes, 3:1); IR (film) $v_{\max } 3354,2919,1731,1649,1602,1484,1437,1372,1343$, 1243, 1208, 1120, 1049, 750, $691 \mathrm{~cm}^{-1}$; ${ }^{1} \mathrm{H}$ NMR $\left(500 \mathrm{MHz}, \mathrm{CDCl}_{3}, 1: 1\right.$ mixture of diastereomers) $\delta 7.41-7.34(\mathrm{~m}, 5 \mathrm{H}), 7.18-7.13(\mathrm{~m}, 3 \mathrm{H}), 7.01(\mathrm{~m}, 1 \mathrm{H}), 6.97(\mathrm{dd}, J=5.2,1.8$ $\mathrm{Hz}, 1 \mathrm{H}), 6.96$ (dd, $J=5.2,1.8 \mathrm{~Hz}, 1 \mathrm{H}), 6.90$ (d, $J=4.0 \mathrm{~Hz}, 1 \mathrm{H}), 6.89$ (d, J=4.0 Hz, $1 \mathrm{H}), 6.79$ $(\mathrm{t}, J=7.4 \mathrm{~Hz}, 1 \mathrm{H}), 6.77(\mathrm{t}, J=7.4 \mathrm{~Hz}, 1 \mathrm{H}), 6.24(\mathrm{~s}, 1 \mathrm{H}), 5.92(\mathrm{t}, J=6.6 \mathrm{~Hz}, 1 \mathrm{H}), 5.30(\mathrm{br} \mathrm{s}, 1$ H), $4.91(\mathrm{dd}, J=12.8,5.9 \mathrm{~Hz}, 1 \mathrm{H}), 4.89(\mathrm{dd}, J=12.8,5.9 \mathrm{~Hz}, 1 \mathrm{H}), 3.74(\mathrm{~s}, 3 \mathrm{H}), 3.68(\mathrm{~s}, 3 \mathrm{H})$, $3.15(\mathrm{~m}, 2 \mathrm{H}), 2.03$ (s, $3 \mathrm{H}), 1.97(\mathrm{~s}, 3 \mathrm{H}) ;{ }^{13} \mathrm{C} \mathrm{NMR}\left(150 \mathrm{MHz}, \mathrm{CDCl}_{3}\right) \delta$ 172.2, $169.7(2 \mathrm{C})$, 
159.2, 147.3 (2 C), 142.4 (2 C), 133.2, 133.0, 131.9, 131.7, 131.3, 129.8, 129.1 (2 C), 128.9, 127.9 (2 C), 127.8, 125.9, 125.8, 123.7, 123.6, 121.3, 110.3 (2 C), 105.2 (2 C), 103.0, 66.8, 53.5, 53.4, 52.6, 52.5, 37.4, 37.3, 23.4, 23.3; HRMS (MALDI-FTMS) calcd for $\mathrm{C}_{26} \mathrm{H}_{23} \mathrm{BrN}_{2} \mathrm{O}_{4} \mathrm{H}^{+}[\mathrm{M}+$ $\left.\mathrm{H}^{+}\right]$507.0914, found 507.0908.

Oxazole 12. L-Serine methyl ester $(214.7 \mathrm{~g}, 138 \mathrm{mmol}, 1.0$ equiv $), \mathrm{EDC} \cdot \mathrm{H}_{2} \mathrm{O}(278 \mathrm{~g}$, $145 \mathrm{mmol}, 1.05$ equiv), and $\mathrm{HOBt} \cdot \mathrm{H}_{2} \mathrm{O}(223 \mathrm{~g}, 145 \mathrm{mmol}, 1.05$ equiv) were added sequentially to a solution of Boc-protected L-valine (300 g, $138 \mathrm{mmol}, 1.0$ equiv) in $\mathrm{CH}_{2} \mathrm{Cl}_{2}(2.5 \mathrm{~L})$ at $25^{\circ} \mathrm{C}$. The resultant mixture was stirred for 5 minutes at $25{ }^{\circ} \mathrm{C}$, and then $\mathrm{Et}_{3} \mathrm{~N}(386 \mathrm{~mL}, 276 \mathrm{mmol}, 2.0$ equiv) was added. After stirring for 12 hours at $25{ }^{\circ} \mathrm{C}$, the reaction mixture was poured into water $(500 \mathrm{~mL})$ and extracted with $\mathrm{CH}_{2} \mathrm{Cl}_{2}(2 \times 300 \mathrm{~mL})$. The combined organic layers were then washed with water $(3 \times 500 \mathrm{~mL})$, dried $\left(\mathrm{MgSO}_{4}\right)$, and concentrated. The resultant product was purified by flash column chromatography ( silica gel, EtOAc/hexanes, 1:1) to give amide 30 (404 g, 92\% yield) as a light yellow solid. Next, Burgess reagent (3.57 g, 15.0 mmol, 1.5 equiv) was added to a solution of a portion of this amide intermediate (3.18 $\mathrm{g}, 10.0 \mathrm{mmol}, 1.0 \mathrm{equiv})$ in THF $(50 \mathrm{~mL})$ at $25^{\circ} \mathrm{C}$, and the resultant mixture was heated to reflux and stirred for 4 hours. Upon completion, the reaction flask contents were poured into water $(50 \mathrm{~mL})$ and extracted with EtOAc $(3 \times 25 \mathrm{~mL})$. The combined organic layers were then washed with water $(50 \mathrm{~mL})$ and brine $(50 \mathrm{~mL})$, dried $\left(\mathrm{MgSO}_{4}\right)$, and concentrated. The resultant light yellow residue was purified by flash column chromatography (silica gel, EtOAc/hexanes, 1:1) to give oxazoline 31 (2.40 g, $80 \%$ yield) as a clear oil. Finally, this new intermediate (2.40 g, $8.0 \mathrm{mmol}, 1.0$ equiv) was dissolved in $\mathrm{CH}_{2} \mathrm{Cl}_{2}(150 \mathrm{~mL})$ and treated sequentially at $0{ }^{\circ} \mathrm{C}$ with $\mathrm{DBU}(1.32 \mathrm{~mL}, 8.8 \mathrm{mmol}$, 1.1 equiv) and a solution of $\mathrm{BrCCl}_{3}\left(0.87 \mathrm{~mL}, 8.8 \mathrm{mmol}, 1.1\right.$ equiv) in $\mathrm{CH}_{2} \mathrm{Cl}_{2}(50 \mathrm{~mL})$. The resultant mixture was then warmed to $25{ }^{\circ} \mathrm{C}$ and stirred for 3 hours. Upon completion, the reaction flask contents were poured into water $(50 \mathrm{~mL})$ and extracted with EtOAc $(3 \times 25 \mathrm{~mL})$. The combined organic layers were then washed with water $(50 \mathrm{~mL})$ and brine $(50 \mathrm{~mL})$, dried $\left(\mathrm{MgSO}_{4}\right)$, and concentrated. The resultant light yellow residue was purified by flash column chromatography (silica gel, EtOAc/hexanes, 1:1) to give oxazole 12 (2.15 g, 90\% yield) as a 
clear oil. 12: $\mathrm{R}_{f}=0.48$ (silica gel, EtOAc/hexanes, 1:1); IR (film) $v_{\max } 3322,2968,1712,1583$, 1520, 1453, 1367, 1322, 1245, 1169, 1109, $1000 \mathrm{~cm}^{-1} ;{ }^{1} \mathrm{H}$ NMR (500 MHz, $\left.\mathrm{CDCl}_{3}\right) \delta 8.17(\mathrm{~s}, 1$ H), $5.30(\mathrm{~d}, J=9.2 \mathrm{~Hz}, 1 \mathrm{H}), 4.77(\mathrm{dd}, J=9.2,5.9 \mathrm{~Hz}, 1 \mathrm{H}), 3.88(\mathrm{~s}, 3 \mathrm{H}), 2.16(\mathrm{dq}, J=5.8,5.5$ $\mathrm{Hz}, 1 \mathrm{H}), 1.40$ (s, $9 \mathrm{H}), 0.89$ (app t, $J=8.3 \mathrm{~Hz}, 6 \mathrm{H}) ;{ }^{13} \mathrm{C} \mathrm{NMR}\left(125 \mathrm{MHz}, \mathrm{CDCl}_{3}\right) \delta 165.6$, 162.0, 155.8, 144.3, 133.5, 80.4, 54.7, 52.6, 33.3, 28.7, 19.1, 18.4; HRMS (MALDI-FTMS) calcd for $\mathrm{C}_{14} \mathrm{H}_{22} \mathrm{~N}_{2} \mathrm{O}_{5} \mathrm{Na}^{+}\left[\mathrm{M}+\mathrm{Na}^{+}\right]$321.1421, found 321.1425.

TFA salt of Boc-protected tryptamine (6). Saturated aqueous $\mathrm{NaHCO}_{3}(15 \mathrm{~mL})$ and $\mathrm{Boc}_{2} \mathrm{O}(2.76 \mathrm{~g}, 12.68 \mathrm{mmol}, 1.5$ equiv) were added sequentially to a solution of tryptamine (1.36 g, $8.5 \mathrm{mmol}, 1.0$ equiv) in 1,4-dioxane $(30 \mathrm{~mL})$ at $25^{\circ} \mathrm{C}$. After stirring the resultant suspension for 2 hours at $25^{\circ} \mathrm{C}$, the reaction flask contents were poured into water $(100 \mathrm{~mL})$ and extracted with EtOAc $(3 \times 150 \mathrm{~mL})$. The combined organic layers were then washed with water $(100 \mathrm{~mL})$ and brine $(100 \mathrm{~mL})$, dried $\left(\mathrm{MgSO}_{4}\right)$, and concentrated. The resultant yellow residue was purified by flash column chromatography (silica gel, EtOAc/hexanes, 1:1) to give the desired Bocprotected intermediate $(2.03 \mathrm{~g}, 92 \%)$ as an amorphous white solid. This compound (2.03 g, 7.81 mmol, 1.0 equiv) was then dissolved in $\mathrm{THF} / \mathrm{H}_{2} \mathrm{O}(9: 1,110 \mathrm{~mL})$, and DDQ $(3.54 \mathrm{~g}, 15.6 \mathrm{mmol}$, 2.0 equiv) was added at $0{ }^{\circ} \mathrm{C}$, yielding a deep red solution. After 2 hours of stirring at $0{ }^{\circ} \mathrm{C}$, the reaction mixture was poured into EtOAc $(250 \mathrm{~mL})$ and washed extensively with saturated aqueous $\mathrm{NaHCO}_{3}(4 \times 250 \mathrm{~mL})$ to remove any excess DDQ and its colored by-products. The organic layer was then dried $\left(\mathrm{MgSO}_{4}\right)$ and concentrated to give the desired intermediate ketone (1.86 g, 87\%) as a yellow solid: $\mathrm{R}_{f}=0.19$ (silica gel, EtOAc/hexanes, 1:1); IR (film) $\mathrm{v}_{\max } 3331$, $3260,1687,1646,1535,1514,1432,1361,1296,1243,1167,1126,920,732 \mathrm{~cm}^{-1}$; ${ }^{1} \mathrm{H}$ NMR $\left(500 \mathrm{MHz}, \mathrm{DMSO}-d_{6}\right) \delta 8.46(\mathrm{~d}, J=3.3 \mathrm{~Hz}, 1 \mathrm{H}), 8.22(\mathrm{~d}, J=7.3 \mathrm{~Hz}, 1 \mathrm{H}), 7.54(\mathrm{~d}, J=7.3 \mathrm{~Hz}$, $1 \mathrm{H}), 7.28$ (dt, $J=7.3,1.5 \mathrm{~Hz}, 1 \mathrm{H}), 7.23(\mathrm{dt}, J=7.3,1.5 \mathrm{~Hz}, 1 \mathrm{H}), 7.07$ (t, $J=5.9 \mathrm{~Hz}, 1 \mathrm{H}), 4.36$ $(\mathrm{d}, J=5.9 \mathrm{~Hz}, 2 \mathrm{H}), 2.56(\mathrm{~m}, 1 \mathrm{H}), 1.47(\mathrm{~s}, 9 \mathrm{H}) ;{ }^{13} \mathrm{C}$ NMR $\left(125 \mathrm{MHz}, \mathrm{DMSO}-d_{6}\right) \delta 191.7$, $156.8,137.2$, 134.2, 126.2, 123.7, 122.6, 122.0, 114.8, 113.0, 78.7, 47.7, 29.1; HRMS (MALDIFTMS) calcd for $\mathrm{C}_{15} \mathrm{H}_{18} \mathrm{~N}_{2} \mathrm{O}_{3} \mathrm{Na}^{+}\left[\mathrm{M}+\mathrm{Na}^{+}\right]$297.1210, found 297.1213. When this fragment was needed for coupling, a portion of the ketone intermediate $(0.074 \mathrm{~g}, 0.274 \mathrm{mmol}, 1.0$ equiv $)$ 
was treated with neat TFA $(5 \mathrm{~mL})$ at $25^{\circ} \mathrm{C}$. After stirring the resultant solution for 10 minutes at $25^{\circ} \mathrm{C}$, the solvent was removed in vacuo (using several benzene co-evaporations to ensure the complete removal of all TFA) to give the keto tryptamine TFA salt 6 (0.069 g, 99\% yield) as a white solid which was used immediately.

MOM-protected 7-bromoisatin (13). Vinyl magnesium bromide $(800 \mathrm{~mL}, 1.0 \mathrm{M}$ in THF, $800 \mathrm{mmol}, 3.0$ equiv) was added quickly via cannula to a solution of 2-bromonitrobenzene (53.9 g, $266.7 \mathrm{mmol}, 1.0$ equiv) in THF $(800 \mathrm{~mL})$ at $-45^{\circ} \mathrm{C}$. After stirring the resultant yellow solution for 45 minutes at $-45{ }^{\circ} \mathrm{C}$, the reaction was quenched by the addition of saturated aqueous $\mathrm{NH}_{4} \mathrm{Cl}(50 \mathrm{~mL})$ and the $\mathrm{pH}$ was then adjusted to a value of 6 by the careful addition of 1 $\mathrm{N}$ aqueous $\mathrm{HCl}$. The resultant biphasic system was extracted with EtOAc $(3 \times 200 \mathrm{~mL})$. The combined organic layers were then washed with water $(200 \mathrm{~mL})$ and brine $(200 \mathrm{~mL})$, dried $\left(\mathrm{MgSO}_{4}\right)$, and concentrated. The resultant brown residue was purified by flash column chromatography (silica gel, hexanes/EtOAc, $1: 0 \rightarrow 1: 8$ ) to give 7-bromoindole (33.0 g, 63\% yield) as a yellow-orange solid. This newly-formed product (33.0 g, $168.4 \mathrm{mmol}, 1.0$ equiv) was then dissolved in DMF (150 mL) and freshly ground $\mathrm{KOH}$ (23.4 g, $420.9 \mathrm{mmol}, 2.5$ equiv) was added at $0{ }^{\circ} \mathrm{C}$. The resultant mixture was stirred for 5 minutes at $0{ }^{\circ} \mathrm{C}$, and then a solution of $\mathrm{I}_{2}$ (44.8 g, $176.8 \mathrm{mmol}, 1.05$ equiv) in DMF $(50 \mathrm{~mL})$ was added at $0{ }^{\circ} \mathrm{C}$. After 15 minutes of additional stirring at $0{ }^{\circ} \mathrm{C}$, the reaction mixture was poured into water $(400 \mathrm{~mL})$ and extracted with $\mathrm{Et}_{2} \mathrm{O}(3 \times 200 \mathrm{~mL})$. The combined organic layers were then washed with water $(200 \mathrm{~mL})$ and brine $(200 \mathrm{~mL})$, dried $\left(\mathrm{MgSO}_{4}\right)$, and concentrated to give 3-iodo-7-bromoindole (49.4 $\mathrm{g}$, 91\% yield) as a red solid. Without further purification, this newly-formed intermediate (49.4 g, $153.4 \mathrm{mmol}, 1.0$ equiv) was dissolved in THF $(500 \mathrm{~mL})$, cooled to $-78{ }^{\circ} \mathrm{C}$, and treated with LiHMDS (184 mL, 1.0 M in THF, $184.0 \mathrm{mmol}, 1.2$ equiv). Following 15 minutes of stirring at $78{ }^{\circ} \mathrm{C}, \mathrm{MOMCl}\left(16.1 \mathrm{~mL}, 214.7 \mathrm{mmol}, 1.4\right.$ equiv) was added at $-78^{\circ} \mathrm{C}$, and the reaction mixture was allowed to warm to $25^{\circ} \mathrm{C}$. After 2 hours of additional stirring at $25^{\circ} \mathrm{C}$, the reaction was quenched by the addition of saturated aqueous $\mathrm{NH}_{4} \mathrm{Cl}(100 \mathrm{~mL})$ and extracted with EtOAc $(3 \times$ $200 \mathrm{~mL})$. The combined organic layers were then washed with water $(200 \mathrm{~mL})$ and brine $(200$ 
$\mathrm{mL})$, dried $\left(\mathrm{MgSO}_{4}\right)$, and concentrated to give $N$-MOM-protected 3-iodo-7-bromoindole (54.0 g, 96\% yield) as a brown solid. This crude product (54.0 g, $147.5 \mathrm{mmol}, 1.0$ equiv) was then dissolved in $\mathrm{CH}_{3} \mathrm{CN} / \mathrm{THF} /$ water $(8.3: 1.0: 1.7,660 \mathrm{~mL})$, cooled to $0{ }^{\circ} \mathrm{C}$, and treated sequentially with $\mathrm{RuCl}_{3} \cdot 3 \mathrm{H}_{2} \mathrm{O}\left(3.77 \mathrm{~g}, 14.8 \mathrm{mmol}, 0.1\right.$ equiv) and $\mathrm{NaIO}_{4}(94.7 \mathrm{~g}, 442.68 \mathrm{mmol}, 3.0$ equiv). The resultant suspension was stirred for 30 minutes at $0{ }^{\circ} \mathrm{C}$ before it was filtered through a short pad of silica gel. Following subsequent removal of the residual solvent in vacuo, the residue was taken up in EtOAc $(200 \mathrm{~mL})$ and washed with saturated aqueous $\mathrm{Na}_{2} \mathrm{~S}_{2} \mathrm{O}_{3}(50 \mathrm{~mL})$, water $(50$ $\mathrm{mL})$, and brine $(50 \mathrm{~mL})$, dried $\left(\mathrm{MgSO}_{4}\right)$, and concentrated. The resultant dark red residue was purified by flash column chromatography (silica gel, EtOAc/hexanes, 0:1 $\rightarrow 40: 1$ ) to give the desired 7-bromoisatin 7 (27.2 g, 68\% yield over 3 steps) as a red-orange solid. $7: \mathrm{R}_{f}=0.42$ (silica gel, EtOAc/hexanes, 1:3); IR (film) $v_{\max } 1737,1608,1461,1426,1337,1284,1173,1085$, 1008, $773 \mathrm{~cm}^{-1} ;{ }^{1} \mathrm{H}$ NMR $\left(600 \mathrm{MHz}, \mathrm{CDCl}_{3}\right) \delta 7.75(\mathrm{~d}, J=7.5 \mathrm{~Hz}, 1 \mathrm{H}), 7.62(\mathrm{~d}, J=7.5 \mathrm{~Hz}, 1$ $\mathrm{H}), 7.05(\mathrm{t}, J=7.5 \mathrm{~Hz}, 1 \mathrm{H}), 5.50(\mathrm{~s}, 2 \mathrm{H}), 3.39(\mathrm{~s}, 3 \mathrm{H}) ;{ }^{13} \mathrm{C} \mathrm{NMR}\left(150 \mathrm{MHz}, \mathrm{CDCl}_{3}\right) \delta 182.2$, 159.2, 147.5, 144.3, 125.7, 124.9, 120.8, 105.4, 71.5, 56.8; HRMS (MALDI-FTMS) calcd for $\mathrm{C}_{10} \mathrm{H}_{8} \mathrm{BrNO}_{3} \mathrm{Na}^{+}\left[\mathrm{M}+\mathrm{Na}^{+}\right] 291.9580$, found 291.9587.

Benzylated oxazole 36. $\mathrm{LiBH}_{4}(301.6 \mathrm{~mL}, 2.0 \mathrm{M}$ in THF, $603.3 \mathrm{mmol}, 3.0$ equiv) and absolute EtOH (58.6 mL, $1005 \mathrm{mmol}, 5.0$ equiv) were added sequentially to a solution of oxazole $12\left(60.0 \mathrm{~g}, 201.1 \mathrm{mmol}, 1.0\right.$ equiv) in $\mathrm{THF}(500 \mathrm{ml})$ at $0{ }^{\circ} \mathrm{C}$. The reaction mixture was then warmed to $25^{\circ} \mathrm{C}$ and allowed to stir for 3 hours. EtOAc (200 mL) was then added dropwise at $25^{\circ} \mathrm{C}$, and after an additional 30 minutes had transpired, the reaction mixture was cooled to $0{ }^{\circ} \mathrm{C}$ and quenched by the careful addition of $1 \mathrm{~N}$ aqueous $\mathrm{HCl}(\sim 300 \mathrm{~mL})$ until the evolution of hydrogen gas ceased, poured into water $(200 \mathrm{~mL})$, and extracted with EtOAc $(3 \times$ $300 \mathrm{~mL})$. The combined organic layers were then washed with water $(200 \mathrm{~mL})$ and brine $(200$ $\mathrm{mL})$, dried $\left(\mathrm{MgSO}_{4}\right)$, and concentrated. The resultant yellow residue was purified by flash column chromatography (silica gel, $\mathrm{CH}_{2} \mathrm{Cl}_{2} /$ EtOAc, $1: 0 \rightarrow 2: 1$ ) to give the desired intermediate alcohol (45.0 g, 83\% yield) as a transparent oil: $\mathrm{R}_{f}=0.43$ (silica gel, EtOAc/hexanes, $\left.3: 1\right) ;[\alpha]_{\mathrm{D}}{ }^{20}$ $=-33.7(c=1.0, \mathrm{MeOH}) ; \mathrm{IR}($ film $) v_{\max } 3307,2954,2872,1701,1519,1464,1367,1247,1170$, 
$1040 \mathrm{~cm}^{-1} ;{ }^{1} \mathrm{H}$ NMR $\left(500 \mathrm{MHz}, \mathrm{CDCl}_{3}\right) \delta 7.51(\mathrm{~s}, 1 \mathrm{H}), 5.58(\mathrm{~d}, J=8.0 \mathrm{~Hz}, 1 \mathrm{H}), 4.71(\mathrm{dd}, J=$ 8.0, $5.5 \mathrm{~Hz}, 1 \mathrm{H}), 4.53(\mathrm{~s}, 2 \mathrm{H}), 4.43$ (s, $1 \mathrm{H}), 2.10$ (dq, J=5.8, $5.5 \mathrm{~Hz}, 1 \mathrm{H}), 1.41$ (s, $9 \mathrm{H}), 0.89$ $(\mathrm{d}, J=5.8 \mathrm{~Hz}, 3 \mathrm{H}), 0.86(\mathrm{~d}, J=5.8 \mathrm{~Hz}, 3 \mathrm{H}) ;{ }^{13} \mathrm{C} \mathrm{NMR}\left(125 \mathrm{MHz}, \mathrm{CDCl}_{3}\right) \delta 165.3,163.6$, 155.9, 135.4, 80.2, 56.4, 54.8, 33.4, 28.7, 19.1, 18.5; HRMS (MALDI-FTMS) calcd for $\mathrm{C}_{13} \mathrm{H}_{22} \mathrm{~N}_{2} \mathrm{O}_{4} \mathrm{Na}^{+}\left[\mathrm{M}+\mathrm{Na}^{+}\right]$293.1472, found 293.1466. Next, NaHMDS (277.4 mL, $1.0 \mathrm{M}$ in THF, $277.4 \mathrm{mmol}, 2.0$ equiv) was added to a solution of this alcohol (50.0 g, $138.7 \mathrm{mmol}, 1.0$ equiv) in THF (1.0 L) at $-78^{\circ} \mathrm{C}$ over the course of 30 minutes. Benzyl bromide $(15.1 \mathrm{~mL}, 138.7$ mmol, 1.0 equiv) and $n$ - $\mathrm{Bu}_{4} \mathrm{NI}\left(0.100 \mathrm{~g}\right.$, catalytic) were then added at $-78^{\circ} \mathrm{C}$, and the resulting mixture was warmed to $25{ }^{\circ} \mathrm{C}$ and stirred for 36 hours. Upon completion, the reaction mixture was poured into brine $(300 \mathrm{~mL})$ and extracted with EtOAc $(3 \times 300 \mathrm{~mL})$. The combined organic layers were then washed with water $(200 \mathrm{~mL})$ and brine $(200 \mathrm{ml})$, dried $\left(\mathrm{MgSO}_{4}\right)$, and concentrated. The resultant yellow residue was purified by flash column chromatography (silica gel, EtOAc/hexanes, 1:20 $\rightarrow 1: 4)$ to give oxazole $36(36.0 \mathrm{~g}, 72 \%$ yield) as a transparent oil. 36: $\mathrm{R}_{f}=0.42$ (silica gel, EtOAc/hexanes, 3:10); $[\alpha]_{\mathrm{D}}{ }^{20}=-26.6(c=1.0, \mathrm{MeOH}) ; \mathrm{IR}\left(\right.$ film) $v_{\max } 3318$, 2954, 1707, 1501, 1448, 1360, 1243, 1167, 1084, $737 \mathrm{~cm}^{-1} ;{ }^{1} \mathrm{H}$ NMR (500 MHz, $\left.\mathrm{CDCl}_{3}\right) \delta 7.51$ (s, $1 \mathrm{H}), 7.33-7.29(\mathrm{~m}, 5 \mathrm{H}), 5.28$ (d, J=7.6 Hz, $1 \mathrm{H}), 4.73$ (dd, $J=7.6,4.7 \mathrm{~Hz}, 1 \mathrm{H}), 4.57$ (s, 2 H), 4.43 (s, $2 \mathrm{H}), 2.16(\mathrm{dq}, J=6.4,4.7 \mathrm{~Hz}, 1 \mathrm{H}), 1.41(\mathrm{~s}, 9 \mathrm{H}), 0.89$ (d, J=6.4 Hz, $3 \mathrm{H}), 0.88$ (d, $J=6.4 \mathrm{~Hz}, 3 \mathrm{H}) ;{ }^{13} \mathrm{C} \mathrm{NMR}\left(125 \mathrm{MHz}, \mathrm{CDCl}_{3}\right) \delta 164.6,163.5,155.8,138.1,136.4,128.8,128.3$, 80.1, 72.9, 64.3, 54.7, 33.3, 28.7, 19.0, 18.3; HRMS (MALDI-FTMS) calcd for $\mathrm{C}_{28} \mathrm{H}_{28} \mathrm{~N}_{2} \mathrm{O}_{4} \mathrm{Na}^{+}$ $\left[\mathrm{M}+\mathrm{Na}^{+}\right]$383.1941, found 383.1938.

Tertiary alcohol 11. To a solution of oxazole 36 (20.0 g, $55.5 \mathrm{mmol}, 1.0$ equiv) in THF $(300 \mathrm{~mL})$ at $-78^{\circ} \mathrm{C}$ was added $n$-BuLi $(69.3 \mathrm{~mL}, 1.6 \mathrm{M}$ in hexanes, $111.0 \mathrm{mmol}, 2.0$ equiv) over the course of 5 minutes, during which time the initial pale yellow color of the solution deepened to a dark brown. After 30 minutes of further stirring at $-78^{\circ} \mathrm{C}$, a solution of isatin derivative $\mathbf{1 3}$ (15.7 g, $58.3 \mathrm{mmol}, 1.05$ equiv) in THF (60 mL) was then added dropwise via cannula over the course of 5 minutes. Upon completion, the reaction mixture was quenched immediately with saturated aqueous $\mathrm{NH}_{4} \mathrm{Cl}(200 \mathrm{~mL})$ and extracted with EtOAc $(3 \times 200 \mathrm{~mL})$. The combined 
organic layers were then washed with water $(200 \mathrm{~mL})$ and brine $(200 \mathrm{~mL})$, dried $\left(\mathrm{MgSO}_{4}\right)$, and concentrated. The resultant orange residue was purified by flash column chromatography (silica gel, hexanes/EtOAc, 10:1 $\rightarrow 2: 1)$ to give tertiary alcohol 11 (25.5 g, 73\% yield) as a yellow foam. 11: $\mathrm{R}_{f}=0.65$ (silica gel, EtOAc/hexanes, 1:1); IR (film) $v_{\max } 3330,2966,2931,2872,1707$, 1607, 1501, 1367, 1172, 1090, $744 \mathrm{~cm}^{-1} ;{ }^{1} \mathrm{H} \mathrm{NMR}\left(400 \mathrm{MHz}, \mathrm{CDCl}_{3}\right) \delta 7.50(\mathrm{~d}, J=8.2 \mathrm{~Hz}, 1$ H), $7.49(\mathrm{~d}, J=8.2 \mathrm{~Hz}, 1 \mathrm{H}), 7.40-7.25(\mathrm{~m}, 12 \mathrm{H}), 6.95$ (dd, $J=8.2,7.4 \mathrm{~Hz}, 1 \mathrm{H}), 6.94(\mathrm{dd}, J=$ 8.2, 7.6 Hz, $1 \mathrm{H}), 6.17$ (s, $1 \mathrm{H}), 6.11(\mathrm{~s}, 1 \mathrm{H}), 5.48$ (d, J=10.8 Hz, $1 \mathrm{H}), 5.45$ (s, $2 \mathrm{H}), 5.42$ (d, $J$ $=10.8 \mathrm{~Hz}, 1 \mathrm{H}), 5.12(\mathrm{~d}, J=9.1 \mathrm{~Hz}, 1 \mathrm{H}), 5.11(\mathrm{~d}, J=8.8 \mathrm{~Hz}, 1 \mathrm{H}), 4.81-4.52(\mathrm{~m}, 10 \mathrm{H}), 3.37$ $(\mathrm{s}, 3 \mathrm{H}), 3.36(\mathrm{~s}, 3 \mathrm{H}), 2.00-1.97(\mathrm{~m}, 2 \mathrm{H}), 1.40$ (s, $9 \mathrm{H}), 1.39(\mathrm{~s}, 9 \mathrm{H}), 0.82-0.77(\mathrm{~m}, 12 \mathrm{H}) ;{ }^{13} \mathrm{C}$ NMR (100 MHz, $\left.\mathrm{CDCl}_{3}\right) \delta 177.0,175.9,162.6$ (2 C), 155.2 (2 C), 145.2, 145.1, 139.6, 139.6, 136.4, 136.2, 135.7, 135.5, 131.3, 131.1, 128.5 (2 C), 128.3 (2 C), 128.2 (2 C), 125.7 (2 C), 125.0, 124.9, 124.5, 124.4, 103.6 (2 C), 79.5 (2 C), 73.3 (2 C), 73.2, 73.1, 71.5 (2 C), 65.2, 65.1, 56.2, 56.1, 54.0, 53.8, 32.7 (2 C), 28.2 (2 C), 18.4, 18.3, 17.8, 17.6; HRMS (MALDI-FTMS) calcd for $\mathrm{C}_{30} \mathrm{H}_{36} \mathrm{BrN}_{3} \mathrm{O}_{7} \mathrm{Na}^{+}\left[\mathrm{M}+\mathrm{Na}^{+}\right]$652.1629, found 652.1634.

Triaryl intermediate 8. Cbz-protected L-tyrosine 9 (41.8 g, $126.8 \mathrm{mmol}, 4.0$ equiv) and p- $\mathrm{TsOH} \cdot \mathrm{H}_{2} \mathrm{O}(24.1 \mathrm{~g}, 126.8 \mathrm{mmol}, 4.0$ equiv) were added sequentially to a solution of tertiary alcohol 11 (20 g, $31.7 \mathrm{mmol}, 1.0$ equiv) in 1,2-dichloroethane at $25{ }^{\circ} \mathrm{C}$. The resultant mixture was then heated to reflux, and after 25 minutes of further stirring, the reaction flask contents were quickly cooled to $0{ }^{\circ} \mathrm{C}$, poured into saturated aqueous $\mathrm{NaHCO}_{3}(300 \mathrm{~mL})$, and extracted with EtOAc $(3 \times 300 \mathrm{~mL})$. The combined organic layers were then washed with water $(3 \times 200$ $\mathrm{mL})$, dried $\left(\mathrm{MgSO}_{4}\right)$, and concentrated. The resultant black residue was purified quickly by flash column chromatography ( silica gel, EtOAc/ $\mathrm{CH}_{2} \mathrm{Cl}_{2}, 0: 1 \rightarrow 3: 1$; then EtOAc/MeOH, 10:1) to give amine 38 (12.0 g, 47\% yield) as a dark foam. This intermediate (12.0 g, $15.0 \mathrm{mmol}, 1.0$ equiv) was then dissolved in 1,4-dioxane $(30 \mathrm{~mL})$, and saturated aqueous $\mathrm{NaHCO}_{3}(15 \mathrm{~mL})$ and $\mathrm{Boc}_{2} \mathrm{O}$ (2.76 g, $12.68 \mathrm{mmol}, 1.5$ equiv) were added sequentially at $25^{\circ} \mathrm{C}$. After stirring the resultant suspension for 2 hours at $25^{\circ} \mathrm{C}$, the reaction flask contents were poured into water $(100 \mathrm{~mL})$ and extracted with EtOAc $(3 \times 150 \mathrm{~mL})$. The combined organic layers were then washed with water 


\section{Revised II}

$(100 \mathrm{~mL})$ and brine $(100 \mathrm{~mL})$, dried $\left(\mathrm{MgSO}_{4}\right)$, and concentrated. The resultant brown residue was purified by flash column chromatography (silica gel, EtOAc/hexanes, 1:20 $\rightarrow 1: 2$ ) to give the desired triaryl product 8 and its diastereomer 39 (4.6 g each, 33\% combined yield over two steps) as pale yellow foams. 8: $\mathrm{R}_{f}=0.38$ (silica gel, EtOAc/hexanes, $\left.1: 1\right) ;[\alpha]_{\mathrm{D}}{ }^{20}=+153.8(c=1.0$, MeOH); IR (film) $v_{\max } 3312,2966,2872,1725,1619,1507,1454,1372,1249,1166,1601,748$ $\mathrm{cm}^{-1} ;{ }^{1} \mathrm{H}$ NMR $\left(600 \mathrm{MHz}, 323 \mathrm{~K}, \mathrm{CD}_{3} \mathrm{CN}\right) \delta 8.78(\mathrm{~s}, 1 \mathrm{H}), 7.82(\mathrm{~s}, 1 \mathrm{H}), 7.43$ (dd, $J=7.9,0.8$ $\mathrm{Hz}, 1 \mathrm{H}), 7.35-7.17(\mathrm{~m}, 10 \mathrm{H}), 7.05$ (dd, $J=7.9,2.2 \mathrm{~Hz}, 1 \mathrm{H}), 6.90$ (dd, $J=8.3,8.2 \mathrm{~Hz}, 1 \mathrm{H})$, $6.78(\mathrm{~d}, J=7.9 \mathrm{~Hz}, 1 \mathrm{H}), 6.77(\mathrm{~d}, J=1.7 \mathrm{~Hz}, 1 \mathrm{H}), 5.86(\mathrm{~s}, 1 \mathrm{H}), 5.63(\mathrm{~s}, 1 \mathrm{H}), 5.04(\mathrm{~d}, J=12.7$ $\mathrm{Hz}, 1 \mathrm{H}), 5.00(\mathrm{~d}, J=12.7 \mathrm{~Hz}, 1 \mathrm{H}), 4.37-4.32(\mathrm{~m}, 1 \mathrm{H}), 4.23(\mathrm{~d}, J=11.8 \mathrm{~Hz}, 1 \mathrm{H}), 4.20(\mathrm{~d}, J=$ $11.8 \mathrm{~Hz}, 1 \mathrm{H}), 4.07$ (d, $J=11.9 \mathrm{~Hz}, 1 \mathrm{H}), 3.94$ (d, $J=11.9 \mathrm{~Hz}, 1 \mathrm{H}), 3.66-3.62(\mathrm{~m}, 1 \mathrm{H}), 3.56$ (s, $3 \mathrm{H}), 2.99(\mathrm{dd}, J=14.2,5.3 \mathrm{~Hz}, 1 \mathrm{H}), 2.80(\mathrm{dd}, J=14.2,8.6 \mathrm{~Hz}, 1 \mathrm{H}), 2.05-2.00$ (m, $1 \mathrm{H}), 1.36$ $(\mathrm{s}, 9 \mathrm{H}), 0.87(\mathrm{~d}, J=6.8 \mathrm{~Hz}, 3 \mathrm{H}), 0.79(\mathrm{~d}, J=6.8 \mathrm{~Hz}, 3 \mathrm{H}) ;{ }^{13} \mathrm{C} \mathrm{NMR}(150 \mathrm{MHz}, 323 \mathrm{~K}$, $\left.\mathrm{CD}_{3} \mathrm{CN}\right) \delta 177.4,172.8,164.1,156.8,156.5,155.2,145.3,141.7,139.3,138.0,135.7,132.8$, $132.1,131.6,130.9,129.4,129.4,129.1,128.8,128.6,128.5,128.3,126.1,126.1,124.9,118.4$, $103.6,80.1,72.7,67.2,64.4,58.1,56.5,55.7,52.6,37.6,32.9,28.5,19.0,18.7$; HRMS (MALDI-FTMS) calcd for $\mathrm{C}_{46} \mathrm{H}_{49} \mathrm{BrN}_{4} \mathrm{O}_{10} \mathrm{Na}^{+}\left[\mathrm{M}+\mathrm{Na}^{+}\right]$919.2524, found 919.2517.

39: $\mathrm{R}_{f}=0.35$ (silica gel, EtOAc/hexanes, $\left.1: 1\right) ;[\alpha]_{\mathrm{D}}{ }^{20}=-119.3(c=0.7, \mathrm{MeOH})$; IR (film) $v_{\max } 3295,2977,2930,1719,1613,1513,1442,1366,1254,1225,1167,1066,749 \mathrm{~cm}^{-1}$; ${ }^{1} \mathrm{H}$ NMR (600 MHz, $\left.323 \mathrm{~K}, \mathrm{CD}_{3} \mathrm{CN}\right) \delta 8.87$ (s, $\left.1 \mathrm{H}\right), 8.10(\mathrm{~s}, 1 \mathrm{H}), 7.41(\mathrm{dd}, J=8.3,0.8 \mathrm{~Hz}, 1$ H), 7.35-7.17 (m, $10 \mathrm{H}), 7.06(\mathrm{dd}, J=7.9,2.2 \mathrm{~Hz}, 1 \mathrm{H}), 6.89$ (dd, $J=8.3,8.3 \mathrm{~Hz}, 1 \mathrm{H}), 6.82$ (d, $J=1.7 \mathrm{~Hz}, 1 \mathrm{H}), 6.78(\mathrm{~d}, J=7.9 \mathrm{~Hz}, 1 \mathrm{H}), 6.01(\mathrm{~s}, 1 \mathrm{H}), 5.71(\mathrm{~s}, 1 \mathrm{H}), 5.05-4.96(\mathrm{~m}, 2 \mathrm{H}), 4.50-$ 4.47 (m, 1 H), 4.37-4.33 (m, $1 \mathrm{H}), 4.20$ (s, $2 \mathrm{H}), 4.01$ (d, J=11.4 Hz, $1 \mathrm{H}), 3.95$ (d, J=11.4 Hz, $1 \mathrm{H}), 3.65-3.60(\mathrm{~m}, 1 \mathrm{H}), 3.61(\mathrm{~s}, 3 \mathrm{H}), 3.03-2.99(\mathrm{~m}, 1 \mathrm{H}), 2.78(\mathrm{dd}, J=14.4,8.8 \mathrm{~Hz}, 1 \mathrm{H})$, 2.03-2.00 (m, $1 \mathrm{H}), 1.37$ (s, $9 \mathrm{H}), 0.87(\mathrm{~d}, J=6.8 \mathrm{~Hz}, 3 \mathrm{H}), 0.79(\mathrm{~d}, J=6.8 \mathrm{~Hz}, 3 \mathrm{H}) ;{ }^{13} \mathrm{C} \mathrm{NMR}$ $\left(150 \mathrm{MHz}, 323 \mathrm{~K}, \mathrm{CD}_{3} \mathrm{CN}\right) \delta 177.2,172.9,164.0,156.9,156.6,155.0,145.2,141.7,139.3$, $138.0,135.7,132.8,131.9,131.4,130.9,129.5,129.3,129.1,128.7,128.6,128.4,128.3,126.2$, $125.1,124.8,118.4,103.5,80.1,72.7,67.0,64.4,58.0,56.6,56.5,52.6,37.6,32.8,28.5,18.9$, 
18.7; HRMS (MALDI-FTMS) calcd for $\mathrm{C}_{46} \mathrm{H}_{49} \mathrm{BrN}_{4} \mathrm{O}_{10} \mathrm{Na}^{+}\left[\mathrm{M}+\mathrm{Na}^{+}\right]$919.2524, found 919.2528.

Bis-MOM protected intermediate $40 . \mathrm{K}_{2} \mathrm{CO}_{3}(31.9 \mathrm{~g}, 230.8 \mathrm{mmol}, 40$ equiv) and MOMCl (8.8 mL, $115.4 \mathrm{mmol}, 20$ equiv) were added sequentially to a solution of phenol 8 (5.17 $\mathrm{g}, 5.77 \mathrm{mmol}, 1.0$ equiv) in acetone $(500 \mathrm{~mL})$ at $0{ }^{\circ} \mathrm{C}$. After 10 minutes of stirring at $0{ }^{\circ} \mathrm{C}$, the reaction mixture was warmed to $25^{\circ} \mathrm{C}$ and stirred for an additional hour. Upon completion, the reaction mixture was filtered through a short pad of $\mathrm{MgSO}_{4}$, concentrated, and purified by flash column chromatography (silica gel, EtOAc/hexanes, 0:1 $\rightarrow 3: 2$ ) to give bis-MOM protected intermediate 40 (4.85 g, 85\% yield) as a pale yellow solid. 40: $\mathrm{R}_{f}=0.42$ (silica gel, EtOAc/hexanes, 1:1); $[\alpha]_{\mathrm{D}}^{20}=-47.6(c=1.0, \mathrm{MeOH})$; IR (film) $v_{\max } 3331,2954,1725,1696$, $1578,1496,1455,1361,1337,1243,1208,1161,1073,996,914,732,697 \mathrm{~cm}^{-1} ;{ }^{1} \mathrm{H}$ NMR (600 $\left.\mathrm{MHz}, \mathrm{CD}_{3} \mathrm{CN}\right) \delta 7.48(\mathrm{~d}, J=7.4 \mathrm{~Hz}, 1 \mathrm{H}), 7.36-7.23(\mathrm{~m}, 10 \mathrm{H}), 7.16(\mathrm{dd}, J=8.3,1.7 \mathrm{~Hz}, 1 \mathrm{H})$, $7.10(\mathrm{~d}, J=7.0 \mathrm{~Hz}, 1 \mathrm{H}), 6.96(\mathrm{~d}, J=8.3 \mathrm{~Hz}, 1 \mathrm{H}), 6.92$ (d, $J=2.2 \mathrm{~Hz}, 1 \mathrm{H}), 6.86$ (t, $J=7.9 \mathrm{~Hz}$, $1 \mathrm{H}), 5.95$ (br s, $1 \mathrm{H}), 5.71($ br s, $1 \mathrm{H}), 5.39\left(\mathrm{AB}, J=10.5 \mathrm{~Hz}, \mathrm{v}_{\mathrm{ab}}=45.1 \mathrm{~Hz}, 2 \mathrm{H}\right), 5.06(\mathrm{AB}, J=$ 12.2 Hz, $\left.v_{\mathrm{ab}}=29.3 \mathrm{~Hz}, 2 \mathrm{H}\right), 4.85\left(\mathrm{AB}, J=6.6 \mathrm{~Hz}, \mathrm{v}_{\mathrm{ab}}=57.4 \mathrm{~Hz}, 2 \mathrm{H}\right), 4.56($ br s, $1 \mathrm{H}), 4.41(\mathrm{~m}$, $1 \mathrm{H}), 4.29\left(\mathrm{AB}, J=11.7 \mathrm{~Hz}, v_{\mathrm{ab}}=11.8 \mathrm{~Hz}, 2 \mathrm{H}\right), 4.05\left(\mathrm{AB}, J=10.6 \mathrm{~Hz}, \mathrm{v}_{\mathrm{ab}}=13.6 \mathrm{~Hz}, 2 \mathrm{H}\right)$, $3.62(\mathrm{~s}, 3 \mathrm{H}), 3.32$ (s, $3 \mathrm{H}), 3.10(\mathrm{dd}, J=13.6,4.9 \mathrm{~Hz}, 1 \mathrm{H}), 3.04$ (s, $3 \mathrm{H}), 2.86(\mathrm{dd}, J=13.6,8.8$ $\mathrm{Hz}, 1 \mathrm{H}), 2.07(\mathrm{~m}, 1 \mathrm{H}), 1.40(\mathrm{~s}, 9 \mathrm{H}), 0.91(\mathrm{~d}, J=6.6 \mathrm{~Hz}, 3 \mathrm{H}), 0.83(\mathrm{~d}, J=6.6 \mathrm{~Hz}, 3 \mathrm{H}) ;{ }^{13} \mathrm{C}$ NMR $\left(150 \mathrm{MHz}, \mathrm{CD}_{3} \mathrm{CN}\right) \delta 176.5,172.8,164.5,153.7,144.2,141.1,139.4,137.0,135.4,133.2$, $131.6,131.2,131.0,129.3,129.0,128.8,128.7,128.6,128.4,128.2,125.7,124.8,115.2,103.3$, 94.7, 72.9, 72.6, 67.1, 64.4, 56.2, 56.1, 52.6, 37.8, 32.7, 28.5, 19.0, 18.7; HRMS (MALDIFTMS) calcd for $\mathrm{C}_{50} \mathrm{H}_{57} \mathrm{BrN}_{4} \mathrm{O}_{12} \mathrm{Na}^{+}\left[\mathrm{M}+\mathrm{Na}^{+}\right]$1007.3048, found 1007.3024.

Macrolactam 42. Solid $\mathrm{LiOH}$ (0.85 g, $20.32 \mathrm{mmol}, 20$ equiv) was added to a solution of 40 (1.0 g, $1.02 \mathrm{mmol})$ in $\mathrm{THF} / \mathrm{CH}_{3} \mathrm{OH} / \mathrm{H}_{2} \mathrm{O}(10: 2: 1,39 \mathrm{~mL})$ at $25{ }^{\circ} \mathrm{C}$ and the resultant mixture was heated at reflux for 20 minutes. The reaction mixture was then poured into EtOAc $(100 \mathrm{~mL})$ and washed with $1 \mathrm{~N}$ aqueous $\mathrm{HCl}(3 \times 25 \mathrm{~mL})$. The combined aqueous layers were then reextracted with EtOAc $(3 \times 25 \mathrm{~mL})$, and the combined organic layers were washed with water 
$(50 \mathrm{~mL})$ and brine $(50 \mathrm{~mL})$, dried $\left(\mathrm{MgSO}_{4}\right)$, and concentrated to the intermediate carboxylic acid (0.961 $\mathrm{g}, 96 \%$ yield) as a white foam. This intermediate $(0.961 \mathrm{~g}, 0.976 \mathrm{mmol}, 1.0$ equiv) was immediately treated with neat TFA $(10 \mathrm{~mL})$ at $25{ }^{\circ} \mathrm{C}$, and after 10 minutes of exposure, the solvent was removed in vacuo (using several benzene co-evaporations to ensure the complete removal of all TFA) to give amino acid 41 (0.820 g, 98\% yield) as a white oil. This newlyformed adduct $\left(0.820 \mathrm{~g}, 0.940 \mathrm{mmol}, 1.0\right.$ equiv) was then dissolved in $\mathrm{CH}_{2} \mathrm{Cl}_{2} / \mathrm{DMF}(2: 1,3.0 \mathrm{~L}$, final concentration of $\left.3 \times 10^{-4} \mathrm{M}\right)$, and HATU (0.857 g, $2.24 \mathrm{mmol}, 2.2$ equiv) and 2,4,6collidine ( $1.14 \mathrm{~mL}, 6.70 \mathrm{mmol}, 6.6$ equiv) were added sequentially at $25^{\circ} \mathrm{C}$. After 12 hours of stirring at $25{ }^{\circ} \mathrm{C}$, the reaction flask contents were concentrated; the residue was taken up in EtOAc $(100 \mathrm{~mL})$ and washed with saturated aqueous $\mathrm{NaHCO}_{3}(2 \times 25 \mathrm{~mL})$. The combined aqueous layers were then reextracted with EtOAc $(2 \times 25 \mathrm{~mL})$, and the combined organic layers were washed with water $(50 \mathrm{~mL})$ and brine $(50 \mathrm{~mL})$, dried $\left(\mathrm{MgSO}_{4}\right)$, and concentrated. The resultant yellow residue was purified by flash column chromatography (silica gel, EtOAc/hexanes, $0: 1 \rightarrow 1: 0)$ to give the bis-MOM protected macrolactam $42(0.140 \mathrm{~g}, 16 \%$ yield $)$ and the mono-MOM protected macrolactam 43 (0.220 g, 27\% yield) as amorphous white solids. 42: $\mathrm{R}_{f}=0.66$ (silica gel, EtOAc/hexanes, $\left.2: 1\right) ;[\alpha]_{\mathrm{D}}{ }^{20}=-177.0(c=0.57, \mathrm{MeOH}) ; \mathrm{IR}\left(\right.$ film) $v_{\max }$ $3295,2931,1791,1666,1531,1496,1455,1331,1243,1155,1073,991,914,732,697 \mathrm{~cm}^{-1} ;{ }^{1} \mathrm{H}$ NMR (600 MHz, CD 3 CN) $\delta 7.53(\mathrm{~d}, J=7.9 \mathrm{~Hz}, 1 \mathrm{H}), 7.38-7.27(\mathrm{~m}, 9 \mathrm{H}), 7.20$ (d, $J=7.4 \mathrm{~Hz}, 2$ H), $7.14(\mathrm{~d}, J=7.0 \mathrm{~Hz}, 2 \mathrm{H}), 6.91(\mathrm{t}, J=8.3 \mathrm{~Hz}, 1 \mathrm{H}), 6.90(\mathrm{~d}, J=8.8 \mathrm{~Hz}, 1 \mathrm{H}), 6.35(\mathrm{~s}, 1 \mathrm{H})$, $6.06(\mathrm{br} \mathrm{d}, 1 \mathrm{H}), 5.37\left(\mathrm{AB}, J=10.1 \mathrm{~Hz}, \mathrm{v}_{\mathrm{ab}}=75.4 \mathrm{~Hz}, 2 \mathrm{H}\right), 5.08\left(\mathrm{AB}, J=12.7 \mathrm{~Hz}, \mathrm{v}_{\mathrm{ab}}=21.0\right.$ $\mathrm{Hz}, 2 \mathrm{H}), 4.91\left(\mathrm{AB}, J=6.5 \mathrm{~Hz}, \mathrm{v}_{\mathrm{ab}}=25.9 \mathrm{~Hz}, 2 \mathrm{H}\right), 4.46(\mathrm{t}, J=7.9 \mathrm{~Hz}, 1 \mathrm{H}), 4.14(\mathrm{t}, J=8.8 \mathrm{~Hz}$, $1 \mathrm{H}), 4.09\left(\mathrm{AB}, J=11.4 \mathrm{~Hz}, v_{\mathrm{ab}}=15.4 \mathrm{~Hz}, 2 \mathrm{H}\right), 3.68\left(\mathrm{AB}, J=11.4 \mathrm{~Hz}, v_{\mathrm{ab}}=25.4 \mathrm{~Hz}, 2 \mathrm{H}\right)$, $3.30(\mathrm{~s}, 3 \mathrm{H}), 3.11(\mathrm{t}, J=12.7 \mathrm{~Hz}, 1 \mathrm{H}), 3.05(\mathrm{~s}, 3 \mathrm{H}), 2.66(\mathrm{~d}, J=12.7 \mathrm{~Hz}, 1 \mathrm{H}), 2.02(\mathrm{~m}, 1 \mathrm{H})$, $1.02(\mathrm{~d}, J=6.1 \mathrm{~Hz}, 3 \mathrm{H}), 0.90(\mathrm{~d}, J=6.1 \mathrm{~Hz}, 3 \mathrm{H}) ;{ }^{13} \mathrm{C} \mathrm{NMR}\left(150 \mathrm{MHz}, \mathrm{CD}_{3} \mathrm{CN}\right) \delta 176.1$, $173.5,164.8,152.8,145.9,141.3,138.9,138.0,136.8,136.0,133.5,130.7,130.5,130.3,130.1$, $129.3,129.2,128.8,128.7,128.6,128.4,125.9,125.0,114.4,103.4,94.2,72.9,72.3,67.0,64.1$, 
57.4, 57.1, 56.3, 55.9, 38.2, 30.7, 19.7, 19.3; HRMS (MALDI-FTMS) calcd for $\mathrm{C}_{44} \mathrm{H}_{45} \mathrm{BrN}_{4} \mathrm{O}_{9} \mathrm{Na}^{+}\left[\mathrm{M}+\mathrm{Na}^{+}\right]$875.2262, found 875.2257.

43: $\mathrm{R}_{f}=0.43$ (silica gel, EtOAc/hexanes, 2:1); IR (film) $v_{\max } 3413,2943,1713,1660$, $1508,1455,1337,1284,1243,1214,1173,1090,1087,750,697 \mathrm{~cm}^{-1} ;{ }^{1} \mathrm{H}$ NMR (600 MHz, $\left.\mathrm{CD}_{3} \mathrm{CN}\right) \delta 7.53(\mathrm{~d}, J=8.3 \mathrm{~Hz}, 1 \mathrm{H}), 7.38-7.27(\mathrm{~m}, 9 \mathrm{H}), 7.20(\mathrm{~d}, J=7.0 \mathrm{~Hz}, 2 \mathrm{H}), 7.13(\mathrm{~d}, J=$ $7.4 \mathrm{~Hz}, 1 \mathrm{H}), 7.03$ (br d, $J=7.4 \mathrm{~Hz}, 1 \mathrm{H}), 6.91$ (t, $J=7.5 \mathrm{~Hz}, 1 \mathrm{H}), 6.69$ (d, J= $7.9 \mathrm{~Hz}, 1 \mathrm{H})$, $6.26(\mathrm{~s}, 1 \mathrm{H}), 6.07(\mathrm{br} \mathrm{d}, 1 \mathrm{H}), 5.34\left(\mathrm{AB}, J=11.0 \mathrm{~Hz}, \mathrm{v}_{\mathrm{ab}}=15.8 \mathrm{~Hz}, 2 \mathrm{H}\right), 5.07(\mathrm{AB}, J=12.3 \mathrm{~Hz}$, $\left.v_{\mathrm{ab}}=22.0 \mathrm{~Hz}, 2 \mathrm{H}\right), 4.45(\mathrm{t}, J=7.5 \mathrm{~Hz}, 1 \mathrm{H}), 4.10(\mathrm{t}, J=7.5 \mathrm{~Hz}, 1 \mathrm{H}), 4.08\left(\mathrm{AB}, J=4.4 \mathrm{~Hz}, \mathrm{v}_{\mathrm{ab}}\right.$ $=16.2 \mathrm{~Hz}, 2 \mathrm{H}), 3.63\left(\mathrm{AB}, J=11.4 \mathrm{~Hz}, \mathrm{v}_{\mathrm{ab}}=22.8 \mathrm{~Hz}, 2 \mathrm{H}\right), 3.31(\mathrm{~s}, 3 \mathrm{H}), 3.08(\mathrm{t}, J=11.8 \mathrm{~Hz}, 1$ H), $2.62(\mathrm{~d}, J=11.8 \mathrm{~Hz}, 1 \mathrm{H}), 2.00(\mathrm{~m}, 1 \mathrm{H}), 1.01(\mathrm{~d}, J=5.7 \mathrm{~Hz}, 3 \mathrm{H}), 0.89$ (d, $J=6.1 \mathrm{~Hz}, 3 \mathrm{H})$; ${ }^{13} \mathrm{C}$ NMR $\left(150 \mathrm{MHz}, \mathrm{CD}_{3} \mathrm{CN}\right) \delta 176.2,173.5,164.7,156.2,152.8,146.3,141.3,138.9,138.0$, $136.6,136.0,133.6,130.8,130.6,129.3,129.2$, 128.8, 128.7, 128.6, 128.4, 127.6, 125.9, 124.9, $116.2,103.2,72.9,72.4,67.0,64.1,57.6,57.0,56.5,38.3,30.7,19.7,19.3$; HRMS (MALDIFTMS) calcd for $\mathrm{C}_{42} \mathrm{H}_{41} \mathrm{BrN}_{4} \mathrm{O}_{8} \mathrm{Na}^{+}\left[\mathrm{M}+\mathrm{Na}^{+}\right]$831.2000, found 831.2001.

Deprotected macrolactam 7. $\mathrm{BCl}_{3}\left(7.6 \mathrm{~mL}, 1.0 \mathrm{M}\right.$ in $\mathrm{CH}_{2} \mathrm{Cl}_{2}, 7.6 \mathrm{mmol}, 20$ equiv) was added dropwise to a solution of bis-MOM protected macrolactam $43(0.325 \mathrm{~g}, 0.38 \mathrm{mmol}, 1.0$ equiv) in $\mathrm{CH}_{2} \mathrm{Cl}_{2}(20 \mathrm{~mL})$ at $-78{ }^{\circ} \mathrm{C}$ over the course of 2 minutes. After 2 hours of additional stirring at $-78{ }^{\circ} \mathrm{C}$, the reaction was quenched by the addition of saturated aqueous $\mathrm{NaHCO}_{3}(20$ $\mathrm{mL})$ and then enough THF was added to generate a biphasic system. Aqueous $3 \mathrm{~N} \mathrm{NaOH}(4 \mathrm{~mL})$ was added at $25^{\circ} \mathrm{C}$ and the mixture was stirred for an additional hour. The resultant mixture was poured into water $(50 \mathrm{~mL})$ and extracted with EtOAc $(2 \times 25 \mathrm{~mL})$. The residual aqueous layer was acidified with $1 \mathrm{~N}$ aqueous $\mathrm{HCl}$ and reextracted with EtOAc $(2 \times 25 \mathrm{~mL})$, and the combined organic layers were then washed with water $(50 \mathrm{~mL})$ and brine $(50 \mathrm{~mL})$, dried $\left(\mathrm{MgSO}_{4}\right)$, and concentrated. The resultant light yellow residue was purified by flash column chromatography (silica gel, $\left.\mathrm{CH}_{2} \mathrm{Cl}_{2} / \mathrm{MeOH}, 1: 0 \rightarrow 9: 1\right)$ to give the deprotected macrolactam 7 (0.167 g, 65\% yield) as an off-white solid. 7: $\mathrm{R}_{f}=0.41$ (silica gel, EtOAc); $[\alpha]_{\mathrm{D}}{ }^{20}=-230.3(c=0.31, \mathrm{MeOH})$; IR (film) $v_{\max } 3392,1715,1649,1614,1508,1455,1261,1220,1126,1090,1020,750,597 \mathrm{~cm}^{-1}$; 
${ }^{1} \mathrm{H}$ NMR $\left(600 \mathrm{MHz}, \mathrm{CD}_{3} \mathrm{CN}\right) \delta 8.66(\mathrm{~s}, 1 \mathrm{H}), 7.47(\mathrm{~d}, J=8.3 \mathrm{~Hz}, 1 \mathrm{H}), 7.37-7.32(\mathrm{~m}, 5 \mathrm{H}), 7.27$ (br s, $1 \mathrm{H}), 7.22(\mathrm{~s}, 1 \mathrm{H}), 7.07$ (d, $J=7.4 \mathrm{~Hz}, 1 \mathrm{H}), 7.02(\mathrm{~d}, J=7.9 \mathrm{~Hz}, 1 \mathrm{H}), 6.90$ (t, $J=7.9 \mathrm{~Hz}$, $1 \mathrm{H}), 6.70(\mathrm{~d}, J=7.9 \mathrm{~Hz}, 1 \mathrm{H}), 6.23(\mathrm{~s}, 1 \mathrm{H}), 6.03(\mathrm{br} \mathrm{d}, J=6.3, \mathrm{~Hz} 1 \mathrm{H}), 5.07(\mathrm{AB}, J=12.3 \mathrm{~Hz}$, $\left.\mathrm{v}_{\mathrm{ab}}=21.9 \mathrm{~Hz}, 2 \mathrm{H}\right), 4.45(\mathrm{t}, J=7.9 \mathrm{~Hz}, 1 \mathrm{H}), 4.11(\mathrm{t}, J=8.3 \mathrm{~Hz}, 1 \mathrm{H}), 3.63(\mathrm{ABX}, J=10.1,5.7$ $\left.\mathrm{Hz}, \mathrm{v}_{\mathrm{ab}}=15.8 \mathrm{~Hz}, 2 \mathrm{H}\right), 3.06(\mathrm{t}, J=12.7 \mathrm{~Hz}, 1 \mathrm{H}), 2.78(\mathrm{br} \mathrm{t}, J=5.6 \mathrm{~Hz}, 1 \mathrm{H}), 2.62(\mathrm{~d}, J=12.7$ $\mathrm{Hz}, 1 \mathrm{H}), 2.00(\mathrm{~m}, 1 \mathrm{H}), 1.01(\mathrm{~d}, J=6.1 \mathrm{~Hz}, 3 \mathrm{H}), 0.91(\mathrm{~d}, J=6.1 \mathrm{~Hz}, 3 \mathrm{H}) ;{ }^{13} \mathrm{C} \mathrm{NMR}(150$ $\left.\mathrm{MHz}, \mathrm{CD}_{3} \mathrm{CN}\right) \delta 175.5,173.4,164.6,156.2,153.0,144.7,142.4,139.0,138.0,133.6,133.0$, $130.6,130.0,129.3,129.0,128.7,128.6,127.7,125.4,124.2,116.2,102.9,67.0,57.6,57.0,55.8$, 38.4, 30.7, 19.6, 19.3; HRMS (MALDI-FTMS) calcd for $\mathrm{C}_{33} \mathrm{H}_{31} \mathrm{BrN}_{4} \mathrm{O}_{7} \mathrm{Na}^{+}\left[\mathrm{M}+\mathrm{Na}^{+}\right]$ 697.1268, found 697.1266. Note: use of the same procedure served to convert 42 into 7 in comparable yields; mixtures of $\mathbf{4 2}$ and $\mathbf{4 3}$ could also be transformed into 7 with equal facility with this protocol.

Macrocyclic precursor 46. Boc-protected tertiary alcohol 11 (0.200 g, 0.318 mmol, 1.0 equiv) was treated with neat TFA $(2 \mathrm{~mL})$ at $25{ }^{\circ} \mathrm{C}$. After stirring the resultant solution for 10 minutes at $25^{\circ} \mathrm{C}$, the solvent was removed in vacuo (using several benzene co-evaporations to ensure the complete removal of all TFA) to give amine $44(0.168 \mathrm{~g}, 99 \%$ yield $)$ as a white foam. This intermediate (0.168 g, $0.318 \mathrm{mmol}, 1.0$ equiv) was then dissolved in DMF ( $5 \mathrm{~mL})$ and Cbzprotected L-tyrosine $\left(0.150 \mathrm{~g}, 0.477 \mathrm{mmol}, 1.5\right.$ equiv), $\mathrm{HOBt} \cdot \mathrm{H}_{2} \mathrm{O},(0.073 \mathrm{~g}, 0.477 \mathrm{mmol}, 1.5$ equiv), and $\mathrm{EDC} \cdot \mathrm{H}_{2} \mathrm{O}(0.092 \mathrm{~g}, 0.477 \mathrm{mmol} 1.5$ equiv $)$ were added sequentially at $25^{\circ} \mathrm{C}$. The resultant mixture was cooled to $0{ }^{\circ} \mathrm{C}$, and then $\mathrm{Et}_{3} \mathrm{~N}(0.133 \mathrm{~mL}, 0.953 \mathrm{mmol}, 3.0$ equiv) was added. After 5 minutes of stirring at $0{ }^{\circ} \mathrm{C}$, the reaction mixture was warmed to $25^{\circ} \mathrm{C}$ and stirred for 12 additional hours. Upon completion, the reaction flask contents were poured into saturated aqueous $\mathrm{NaHCO}_{3}(10 \mathrm{~mL})$ and extracted with EtOAc $(3 \times 25 \mathrm{~mL})$. The combined organic layers were then washed with $1 \mathrm{~N}$ aqueous $\mathrm{HCl}(3 \times 10 \mathrm{~mL})$ and brine $(25 \mathrm{~mL})$, dried $\left(\mathrm{MgSO}_{4}\right)$, and concentrated. The resultant yellow oil was purified by flash chromatography (silica gel, $\mathrm{CH}_{2} \mathrm{Cl}_{2} /$ EtOAc, $\left.1: 0 \rightarrow 3: 1\right)$ to give the desired macrocyclic precursor $46(0.167 \mathrm{~g}, 63 \%$ yield $)$ as a yellow foam. 46: $\mathrm{R}_{f}=0.34$ (silica gel, EtOAc/hexanes, 2:1); IR (film) $v_{\max }$ 3307, 3060, 2966, 
$1738,1673,1620,1521,1462,1350,1244,743 \mathrm{~cm}^{-1} ;{ }^{1} \mathrm{H}$ NMR $\left(600 \mathrm{MHz}, \mathrm{CD}_{3} \mathrm{CN}\right) \delta 7.45(\mathrm{t}, J=$ $8.8 \mathrm{~Hz}, 2 \mathrm{H}), 7.40-6.95(\mathrm{~m}, 10 \mathrm{H}), 7.22$ (d, $J=7.0 \mathrm{~Hz}, 4 \mathrm{H}), 7.10(\mathrm{~d}, J=8.3 \mathrm{~Hz}, 1 \mathrm{H}), 7.08(\mathrm{~d}, J$ $=8.3 \mathrm{~Hz}, 1 \mathrm{H}), 7.00-6.90(\mathrm{~m}, 4 \mathrm{H}), 6.64(\mathrm{~d}, J=7.0 \mathrm{~Hz}, 4 \mathrm{H}), 5.96(\mathrm{~d}, J=8.7 \mathrm{~Hz}, 1 \mathrm{H}), 5.95(\mathrm{~d}, J$ $=8.7 \mathrm{~Hz}, 1 \mathrm{H}), 5.59$ (s, $2 \mathrm{H}), 5.35-5.30(\mathrm{~m}, 4 \mathrm{H}), 5.05-4.90(\mathrm{~m}, 4 \mathrm{H}), 4.80-4.70(\mathrm{~m}, 2 \mathrm{H}), 4.50-$ $4.40(\mathrm{~m}, 8 \mathrm{H}), 4.30-4.20(\mathrm{~m}, 2 \mathrm{H}), 3.28(\mathrm{~s}, 3 \mathrm{H}), 3.27$ (s, $3 \mathrm{H}), 2.85(\mathrm{dd}, J=14.0,9.3 \mathrm{~Hz}, 1 \mathrm{H})$, $2.78(\mathrm{dd}, J=14.0,9.3 \mathrm{~Hz}, 1 \mathrm{H}), 2.64(\mathrm{dd}, J=14.0,9.1 \mathrm{~Hz}, 1 \mathrm{H}), 2.56(\mathrm{dd}, J=14.0,9.1 \mathrm{~Hz}, 1 \mathrm{H})$, 2.10-2.00 (m, $2 \mathrm{H}), 0.83(\mathrm{~d}, J=6.6 \mathrm{~Hz}, 3 \mathrm{H}), 0.82(\mathrm{~d}, J=6.6 \mathrm{~Hz}, 3 \mathrm{H}), 0.75(\mathrm{~d}, J=6.6 \mathrm{~Hz}, 3 \mathrm{H})$, $0.72(\mathrm{~d}, J=6.6 \mathrm{~Hz}, 3 \mathrm{H}) ;{ }^{13} \mathrm{C} \mathrm{NMR}\left(150 \mathrm{MHz}, \mathrm{CD}_{3} \mathrm{CN}\right) \delta 176.4,176.3,172.4$ (2 C), 163.1, $163.0,156.9,156.8,156.5,156.4,146.0,145.9,140.4,140.3,138.9$ (2 C), 137.9 (2 C), 137.1, 137.0, 136.1, 136.0, 132.7, 132.6, 129.4 (2 C), 129.3 (4 C), 129.2 (2 C), 129.1 (4 C), 129.0 (2 C), 128.9 (2 C), 128.8 (2 C), 128.7 (2 C), 128.6 (2 C), 128.4 (2 C), 115.9 (2 C), 104.0 (2 C), 74.3, 74.1, 73.1, 73.0, 72.3, 72.2, 67.0, 66.9, 64.8, 64.7, 57.1 (2 C), 56.3 (2 C), 53.7, 53.6, 37.8, 37.7, 19.0, 18.9, 18.6, 18.5; HRMS (MALDI-FTMS) calcd for $\mathrm{C}_{42} \mathrm{H}_{43} \mathrm{BrN}_{4} \mathrm{O}_{9} \mathrm{Na}^{+}\left[\mathrm{M}+\mathrm{Na}^{+}\right]$ 849.2111, found 849.2112.

Boc-protected primary alcohol 47. $\mathrm{Boc}_{2} \mathrm{O}\left(0.061 \mathrm{~g}, 0.28 \mathrm{mmol}, 1.05\right.$ equiv), $\mathrm{Et}_{3} \mathrm{~N}$ (0.081 mL, $0.588 \mathrm{mmol}, 2.2$ equiv) and 4-DMAP (0.003 g, $0.0267 \mathrm{mmol}, 0.1$ equiv) were added sequentially to a solution of deprotected macrolactam 7 ( $0.180 \mathrm{~g}, 0.267 \mathrm{mmol}, 1.0$ equiv) in 1,4dioxane $(5.5 \mathrm{~mL})$ at $25^{\circ} \mathrm{C}$. After 1 hour of stirring at $25{ }^{\circ} \mathrm{C}$, the reaction mixture was diluted with EtOAc $(5 \mathrm{~mL})$, quenched by the addition of $0.5 \mathrm{~N}$ aqueous $\mathrm{HCl}(5 \mathrm{~mL})$, poured into water $(25 \mathrm{~mL})$, and extracted with EtOAc $(3 \times 25 \mathrm{~mL})$. The combined organic layers were then washed with water $(50 \mathrm{~mL})$ and brine $(50 \mathrm{~mL})$, dried $\left(\mathrm{MgSO}_{4}\right)$, and concentrated. The resultant light yellow residue was purified by flash column chromatography (silica gel, $\mathrm{CH}_{2} \mathrm{Cl}_{2} / \mathrm{MeOH}$, $1: 0 \rightarrow 9: 1)$ to give Boc-protected primary alcohol $47(0.145 \mathrm{~g}, 70 \%$ yield $)$ as an off-white solid. 47: $\mathrm{R}_{f}=0.31$ (silica gel, EtOAc/hexanes, 5:1); $[\alpha]_{\mathrm{D}}{ }^{20}=-204.8(c=0.73, \mathrm{MeOH}) ; \mathrm{IR}\left(\right.$ film) $v_{\max }$ $3413,2919,1713,1660,1502,1449,1284,1249,1214,1143,1020,738,650,609 \mathrm{~cm}^{-1} ;{ }^{1} \mathrm{H}$ NMR (600 MHz, CD 3 CN) $\delta 8.86(\mathrm{~s}, 1 \mathrm{H}), 7.49(\mathrm{~d}, J=7.9 \mathrm{~Hz}, 1 \mathrm{H}), 7.38-7.30(\mathrm{~m}, 5 \mathrm{H}), 7.23$ (br d, $1 \mathrm{H}), 7.08(\mathrm{~d}, J=7.4 \mathrm{~Hz}, 1 \mathrm{H}), 7.06(\mathrm{~d}, J=8.3 \mathrm{~Hz}, 1 \mathrm{H}), 6.91(\mathrm{t}, J=7.9 \mathrm{~Hz}, 1 \mathrm{H}), 6.40(\mathrm{~s}, 1$ 
H), 6.08 (br d, $1 \mathrm{H}), 5.08\left(\mathrm{AB}, J=12.3 \mathrm{~Hz}, \mathrm{v}_{\mathrm{ab}}=20.6 \mathrm{~Hz}, 2 \mathrm{H}\right), 4.46(\mathrm{t}, J=7.4 \mathrm{~Hz}, 1 \mathrm{H}), 4.17(\mathrm{t}$, $J=8.8 \mathrm{~Hz}, 1 \mathrm{H}), 3.64\left(\mathrm{AB}, J=12.7 \mathrm{~Hz}, \mathrm{v}_{\mathrm{ab}}=22.8 \mathrm{~Hz}, 2 \mathrm{H}\right), 3.16(\mathrm{t}, J=12.2 \mathrm{~Hz}, 1 \mathrm{H}), 2.82(\mathrm{br}$ s, $1 \mathrm{H}), 2.73(\mathrm{~d}, J=12.2 \mathrm{~Hz}, 1 \mathrm{H}), 2.01(\mathrm{~m}, 1 \mathrm{H}), 1.38(\mathrm{~s}, 9 \mathrm{H}), 1.02(\mathrm{~d}, J=5.7 \mathrm{~Hz}, 3 \mathrm{H}), 0.92$ (d, $J=7.0 \mathrm{~Hz}, 3 \mathrm{H}) ;{ }^{13} \mathrm{C} \mathrm{NMR}\left(150 \mathrm{MHz}, \mathrm{CD}_{3} \mathrm{CN}\right) \delta 174.9,173.2,164.9,151.1,147.2,143.7$, $142.2,139.5,134.8,133.6,133.5,133.2,130.6,129.3$ (2 C), 128.8, 128.6, 125.7, 124.6, 123.6, 103.4, 84.4, 67.0, 57.2, 57.0, 55.8, 38.4, 30.6, 27.4, 19.6, 19.3; HRMS (MALDI-FTMS) calcd for $\mathrm{C}_{38} \mathrm{H}_{39} \mathrm{BrN}_{4} \mathrm{O}_{9} \mathrm{Na}^{+}\left[\mathrm{M}+\mathrm{Na}^{+}\right]$797.1792, found 797.1791.

Amide 5. A solution of IBX (0.174 g, $0.612 \mathrm{mmol}, 5.0$ equiv) in DMSO (2.0 mL) was added to a solution of Boc-protected alcohol 47 (0.095 g, $0.123 \mathrm{mmol}, 1.0$ equiv) in DMSO (1.5 $\mathrm{mL}$ ) at $25^{\circ} \mathrm{C}$. After stirring for 2 hours at $25^{\circ} \mathrm{C}$, the reaction mixture was poured into EtOAc and washed extensively with saturated aqueous $\mathrm{Na}_{2} \mathrm{~S}_{2} \mathrm{O}_{3}(3 \times 25 \mathrm{~mL})$ to remove the IBA byproduct and any residual IBX. The combined organic layers were then washed with water $(3 \times$ $20 \mathrm{~mL})$ and brine $(20 \mathrm{~mL})$, dried $\left(\mathrm{MgSO}_{4}\right)$, and concentrated to give the desired aldehyde $(0.084$ g, 89\% yield) as a yellow foam. Pressing forward without any further purification, this newlyformed intermediate $(0.084 \mathrm{~g}, 0.108 \mathrm{mmol}, 1.0$ equiv) was dissolved in DMSO (3 mL) and treated sequentially at $25{ }^{\circ} \mathrm{C}$ with resorcinol $(0.0671 \mathrm{~g}, 0.613 \mathrm{mmol}, 5.0$ equiv), a solution of $\mathrm{NaH}_{2} \mathrm{PO}_{4} \cdot \mathrm{H}_{2} \mathrm{O}\left(0.094 \mathrm{~g}, 0.613 \mathrm{mmol}, 5.0\right.$ equiv) in water $(0.3 \mathrm{~mL})$, and a solution of $\mathrm{NaClO}_{2}$ (0.045 g, $0.613 \mathrm{mmol}, 5.0$ equiv) in water $(0.3 \mathrm{~mL})$. After stirring for 2 hours at $25^{\circ} \mathrm{C}$, the reaction mixture was poured into saturated aqueous $\mathrm{NH}_{4} \mathrm{Cl}(20 \mathrm{~mL})$ and extracted with EtOAc (3 $\times 25 \mathrm{~mL})$. The combined organic layers were then washed with water $(20 \mathrm{~mL})$ and brine $(20$ $\mathrm{mL})$, dried $\left(\mathrm{MgSO}_{4}\right)$, and concentrated. The resultant residue was purified by flash column chromatography (silica gel, EtOAc/hexanes, $4: 1 \rightarrow 1: 0$; then $\mathrm{MeOH} / \mathrm{EtOAc}, 1: 4 \rightarrow 1: 1$ ) to give the desired free acid $(0.072 \mathrm{~g}, 74 \%$ yield $)$ as a white oil. This intermediate $(0.072 \mathrm{~g}, 0.091 \mathrm{mmol}$, 1.0 equiv) was then dissolved in $\operatorname{DMF}(2.0 \mathrm{~mL})$ and treated sequentially at $25{ }^{\circ} \mathrm{C}$ with freshly prepared keto tryptamine TFA salt 6 (0.074 g, 0.274 mmol, 3.0 equiv), $\mathrm{NaHCO}_{3}(0.122 \mathrm{~g}, 1.37$ mmol, 15 equiv), $\mathrm{EDC} \cdot \mathrm{H}_{2} \mathrm{O}\left(0.053 \mathrm{~g}, 0.274 \mathrm{mmol}, 3.0\right.$ equiv) and $\mathrm{HOBt} \cdot \mathrm{H}_{2} \mathrm{O}(0.043 \mathrm{~g}, 0.274$ mmol, 3.0 equiv). After 12 hours of stirring at $25^{\circ} \mathrm{C}$, the reaction mixture was poured into 
saturated aqueous $\mathrm{NaHCO}_{3}(25 \mathrm{~mL})$ and extracted with EtOAc $(3 \times 25 \mathrm{~mL})$. The combined organic layers were then washed with $1 \mathrm{~N}$ aqueous $\mathrm{HCl}(20 \mathrm{~mL})$, water $(20 \mathrm{~mL})$, and brine $(20$ $\mathrm{mL})$, dried $\left(\mathrm{MgSO}_{4}\right)$, and concentrated. The resultant light yellow residue was purified by flash column chromatography (silica gel, EtOAc/hexanes, $0: 1 \rightarrow 1: 0)$ to give amide $5(0.066 \mathrm{~g}, 76 \%$ yield) as a yellow solid. 5: $\mathrm{R}_{f}=0.36$ (silica gel, EtOAc/hexanes, $\left.1: 1\right) ;[\alpha]_{\mathrm{D}}{ }^{20}=-133.5(c=0.49$, $\mathrm{MeOH}$ ); IR (film) $v_{\max } 3262,1726,1662,1538,1503,1426,1280,1244,1209,1144,1039,751$ $\mathrm{cm}^{-1} ;{ }^{1} \mathrm{H}$ NMR $\left(600 \mathrm{MHz}, \mathrm{CD}_{3} \mathrm{CN}\right) \delta 10.02(\mathrm{~s}, 1 \mathrm{H}), 8.91(\mathrm{~s}, 1 \mathrm{H}), 8.22(\mathrm{~d}, J=7.4 \mathrm{~Hz}, 1 \mathrm{H}), 8.01$ (s, $1 \mathrm{H}), 7.65(\mathrm{~s}, 1 \mathrm{H}), 7.50(\mathrm{~d}, J=7.4 \mathrm{~Hz}, 1 \mathrm{H}), 7.37-7.22(\mathrm{~m}, 10 \mathrm{H}), 7.04$ (br d, $1 \mathrm{H}), 6.96$ (br d, $1 \mathrm{H}), 6.74(\mathrm{t}, J=7.4 \mathrm{~Hz}, 1 \mathrm{H}), 6.58(\mathrm{~s}, 1 \mathrm{H}), 6.25(\mathrm{~d}, J=7.4 \mathrm{~Hz}, 1 \mathrm{H}), 5.10(\mathrm{~m}, 2 \mathrm{H}), 4.57$ (br t, 1 H), $4.35\left(\mathrm{AB}, J=14.6 \mathrm{~Hz}, \mathrm{v}_{\mathrm{ab}}=39.1 \mathrm{~Hz}, 2 \mathrm{H}\right), 4.25$ (br t, $\left.1 \mathrm{H}\right), 3.18(\mathrm{t}, J=11.4 \mathrm{~Hz}, 1 \mathrm{H}), 2.79$ $(\mathrm{d}, J=11.4 \mathrm{~Hz}, 1 \mathrm{H}), 2.05(\mathrm{~m}, 1 \mathrm{H}), 1.40(\mathrm{~s}, 9 \mathrm{H}), 1.03(\mathrm{br} \mathrm{d}, 3 \mathrm{H}), 0.94(\mathrm{br} \mathrm{d}, 3 \mathrm{H}) ;{ }^{13} \mathrm{C}$ NMR $\left(150 \mathrm{MHz}, \mathrm{CD}_{3} \mathrm{CN}\right) \delta 189.7,174.1,173.2,164.1,160.3,156.2,151.2,150.1,146.9,142.4$, $138.0,137.3,134.5,134.2,134.0,133.7,133.0,132.6,131.1,130.5,129.3,128.8,128.6,126.3$, $124.9,124.5,124.2,123.9,123.2,122.3,115.1,112.9,102.8,84.4,67.0,57.2,56.7,46.5,38.3$, 30.6, 27.5, 19.6, 19.3; HRMS (MALDI-FTMS) calcd for $\mathrm{C}_{48} \mathrm{H}_{45} \mathrm{BrN}_{6} \mathrm{O}_{10} \mathrm{Na}^{+}\left[\mathrm{M}+\mathrm{Na}^{+}\right]$ 967.2273, found 967.2269 .

Oxazole 4. $\mathrm{POCl}_{3}(3.0 \mathrm{~mL})$ was added to a solution of amide $5(0.380 \mathrm{~g}, 0.402 \mathrm{mmol}$, 1.0 equiv) in pyridine $(12.0 \mathrm{~mL})$ at $25^{\circ} \mathrm{C}$. After 2 hours of stirring at $25^{\circ} \mathrm{C}$, the reaction mixture was diluted with EtOAc $(10 \mathrm{~mL})$, poured into a cold $\left(0^{\circ} \mathrm{C}\right)$ solution of saturated aqueous $\mathrm{NaHCO}_{3}(50 \mathrm{~mL})$, and extracted with EtOAc $(3 \times 25 \mathrm{~mL})$. The combined organic layers were then washed with water $(50 \mathrm{~mL})$ and brine $(50 \mathrm{~mL})$, dried $\left(\mathrm{MgSO}_{4}\right)$, and concentrated. The resultant light yellow residue was purified by flash column chromatography (silica gel, EtOAc/hexanes, $0: 1 \rightarrow 1: 0)$ to give oxazole $4(0.188 \mathrm{~g}, 52 \%$ yield $)$ as an off-white solid. $4: \mathrm{R}_{f}=$ 0.57 (silica gel, EtOAc/hexanes, 1:1); $[\alpha]_{\mathrm{D}}{ }^{20}=-199.7(c=0.69, \mathrm{MeOH}) ; \mathrm{IR}$ (film) $v_{\max } 3279$, 2927, 1733, 1700, 1652, 1612, 1535, 1506, 1453, 1247, 1212, 1141, 1059, $742 \mathrm{~cm}^{-1} ;{ }^{1} \mathrm{H}$ NMR (600 MHz, CD 3 CN) $\delta 9.86(\mathrm{~s}, 1 \mathrm{H}), 9.20(\mathrm{~s}, 1 \mathrm{H}), 7.69$ (d, J=7.9 Hz, $1 \mathrm{H}), 7.50-7.17$ (m, $11 \mathrm{H})$, 7.08 (s, 2 H), 6.92 (d, J=7.9 Hz, 1 H), 6.68 (d, J= 7.4 Hz, 1 H), 6.43 (m, 2 H), 6.28 (br d, 1 H), 
$5.09\left(\mathrm{AB}, J=12.3 \mathrm{~Hz}, v_{\mathrm{ab}}=22.8 \mathrm{~Hz}, 2 \mathrm{H}\right), 4.60(\mathrm{t}, J=5.7 \mathrm{~Hz}, 1 \mathrm{H}), 4.23(\mathrm{t}, J=8.8 \mathrm{~Hz}, 1 \mathrm{H})$, $3.23(\mathrm{t}, J=11.8 \mathrm{~Hz}, 1 \mathrm{H}), 2.76(\mathrm{~d}, J=11.8 \mathrm{~Hz}, 1 \mathrm{H}), 2.05(\mathrm{~m}, 1 \mathrm{H}), 1.37(\mathrm{~s}, 9 \mathrm{H}), 1.05(\mathrm{~d}, J=5.7$ $\mathrm{Hz}, 3 \mathrm{H}), 0.94(\mathrm{~d}, J=7.0 \mathrm{~Hz}, 3 \mathrm{H}) ;{ }^{13} \mathrm{C} \mathrm{NMR}\left(150 \mathrm{MHz}, \mathrm{CD}_{3} \mathrm{CN}\right) \delta 174.3,173.5,165.8,156.3$, $151.6,151.2,149.7,148.6,147.0,142.4,138.0,137.4,135.0,133.2,132.9,130.8,129.4,129.2$, $129.1,128.8,128.6,124.6$ (2 C), 124.2, 124.1, 123.9, 123.4, 121.7, 121.4, 120.2, 112.8, 104.5, 103.4, 84.5, 67.1, 57.4, 57.2, 57.1, 38.4, 30.7, 27.4, 19.7, 19.3; HRMS (MALDI-FTMS) calcd for $\mathrm{C}_{48} \mathrm{H}_{43} \mathrm{BrN}_{6} \mathrm{O}_{9} \mathrm{H}^{+}\left[\mathrm{M}+\mathrm{H}^{+}\right]$927.2347, found 927.2335.

Wiktop photocyclization product 3. To a solution of oxazole $4(11.8 \mathrm{mg}, 0.013 \mathrm{mmol}$, 1.0 equiv) in $\mathrm{CH}_{3} \mathrm{CN} / \mathrm{H}_{2} \mathrm{O}(3: 1,4.8 \mathrm{~mL})$ at $25^{\circ} \mathrm{C}$ was added $\mathrm{LiOAc} \cdot \mathrm{H}_{2} \mathrm{O}(51.8 \mu \mathrm{L}$ of a solution containing $100 \mathrm{mg}$ in $2.0 \mathrm{~mL}$ of $\mathrm{H}_{2} \mathrm{O}, 0.026 \mathrm{mmol}, 2.0$ equiv) and epichlorohydrin (70.9 $\mu \mathrm{L}$ of a solution containing $100 \mathrm{mg}$ in $2.0 \mathrm{~mL}$ of $\mathrm{CH}_{3} \mathrm{CN}, 0.039 \mathrm{mmol}, 3.0$ equiv). A balloon filled with argon gas and equipped with a long syringe was then inserted into the reaction vessel, and argon gas was bubbled directly into the reaction solution for 7 minutes to remove any dissolved gases. The resulting reaction mixture was exposed to UV light $(200 \mathrm{~nm})$ for 15 minutes at $25^{\circ} \mathrm{C}$. Upon completion, the contents were poured into water $(10 \mathrm{~mL})$ and extracted with EtOAc $(3 \times 25 \mathrm{~mL})$. The combined organic layers were then washed with water $(25 \mathrm{~mL})$ and brine $(25 \mathrm{~mL})$, dried $\left(\mathrm{MgSO}_{4}\right)$, and concentrated. The resultant bright yellow residue was purified by flash column chromatography (silica gel, EtOAc/hexanes, $0: 1 \rightarrow 1: 0$ ) to give the desired photocyclization product 3 (3.6 mg, 33\% yield) as a yellow solid along with a significant portion of recovered starting material (4.2 mg, 51\% yield of $\mathbf{3}$ based on r.s.m.). 3: $\mathrm{R}_{f}=0.25$ (silica gel, EtOAc/hexanes, 5:1); $[\alpha]_{\mathrm{D}}{ }^{20}=-172.26(c=0.31, \mathrm{MeOH}) ; \mathrm{IR}\left(\right.$ film) $v_{\max } 3342,2919,1719$, 1655, 1496, 1443, 1278, 1255, 1143, 1055, $750 \mathrm{~cm}^{-1} ;{ }^{1} \mathrm{H}$ NMR (600 MHz, CD $\left.{ }_{3} \mathrm{CN}\right) \delta 9.87(\mathrm{~s}, 1$ H), $8.68(\mathrm{~s}, 1 \mathrm{H}), 7.60(\mathrm{~d}, J=7.9 \mathrm{~Hz}, 1 \mathrm{H}), 7.43-7.30(\mathrm{~m}, 9 \mathrm{H}), 7.14(\mathrm{~d}, J=6.6 \mathrm{~Hz}, 1 \mathrm{H}), 7.13$ (d, $J=7.9 \mathrm{~Hz}, 1 \mathrm{H}), 6.97(\mathrm{~s}, 1 \mathrm{H}), 6.87-6.86(\mathrm{~m}, 2 \mathrm{H}), 6.83$ (d, $J=7.4 \mathrm{~Hz}, 1 \mathrm{H}), 6.75$ (t, $J=7.0 \mathrm{~Hz}$, $1 \mathrm{H}), 6.24(\mathrm{~d}, J=7.4 \mathrm{~Hz}, 1 \mathrm{H}), 5.09\left(\mathrm{AB}, J=12.7 \mathrm{~Hz}, v_{\mathrm{ab}}=17.1 \mathrm{~Hz}, 2 \mathrm{H}\right), 4.54(\mathrm{t}, J=7.0 \mathrm{~Hz}, 1$ H), $4.31(\mathrm{ddd}, J=11.8,8.3,3.1 \mathrm{~Hz}, 1 \mathrm{H}), 3.32(\mathrm{t}, J=12.3 \mathrm{~Hz}, 1 \mathrm{H}), 2.80(\mathrm{~d}, J=12.7 \mathrm{~Hz}, 1 \mathrm{H})$, $1.98(\mathrm{~m}, 1 \mathrm{H}), 1.41(\mathrm{~s}, 9 \mathrm{H}), 1.00(\mathrm{~d}, J=6.2 \mathrm{~Hz}, 3 \mathrm{H}), 0.90(\mathrm{~d}, J=6.2 \mathrm{~Hz}, 3 \mathrm{H}) ;{ }^{13} \mathrm{C}$ NMR $(150$ 
$\left.\mathrm{MHz}, \mathrm{CD}_{3} \mathrm{CN}\right) \delta 174.4,173.6,164.0,156.3,154.7,151.4,150.4,148.9,147.1,143.3,138.0$, $137.6,134.7,134.6,132.2,131.1,130.9,130.5,129.4,128.8,128.6,128.2,128.0,127.8,126.5$, 124.9, 124.1 (2 C), 123.9, 123.4, 123.2, 121.7, 113.1, 103.5, 84.1, 67.1, 57.5, 57.0, 56.5, 38.0, 31.0, 27.6, 19.7, 19.1; HRMS (MALDI-HRMS) calcd for $\mathrm{C}_{48} \mathrm{H}_{42} \mathrm{~N}_{6} \mathrm{O}_{9} \mathrm{H}^{+}\left[\mathrm{M}+\mathrm{H}^{+}\right]$847.3086, found 847.3110 .

Bis-chlorinated intermediate 49. $N$-chlorosuccinimide $(1.10 \mathrm{~mL}$ of a solution containing $25 \mathrm{mg}$ in $2 \mathrm{~mL}$ of $1: 1 \mathrm{THF} / \mathrm{CCl}_{4}, 0.102 \mathrm{mmol}, 4.4$ equiv) was added to a solution of macrocycle 3 (19.7 mg, $0.023 \mathrm{mmol}, 1.0$ equiv) in $\mathrm{THF} / \mathrm{CCl}_{4}(1: 1,19 \mathrm{~mL})$ at $25{ }^{\circ} \mathrm{C}$. The resulting mixture was then heated at $60{ }^{\circ} \mathrm{C}$ for 2 hours before it was purified directly by preparative TLC (silica gel, EtOAc/hexanes, 5:1) to give the bis-chlorinated product 49 (11.0 $\mathrm{mg}, 50 \%$ yield) as a yellow solid. 49: $\mathrm{R}_{f}=0.60$ (silica gel, EtOAc/hexanes, $5: 1$ ); $[\alpha]_{\mathrm{D}}{ }^{20}=-208.2$ $(c=0.22, \mathrm{MeOH})$; IR (film) $v_{\max } 3260,2966,1725,1666,1502,1443,1273,1243,1208,1143$, 1055, $750 \mathrm{~cm}^{-1} ;{ }^{1} \mathrm{H} \mathrm{NMR}\left(600 \mathrm{MHz}, \mathrm{CD}_{3} \mathrm{CN}\right) \delta 10.4(\mathrm{~s}, 1 \mathrm{H}), 8.62(\mathrm{~s}, 1 \mathrm{H}), 7.55(\mathrm{~d}, J=8.3 \mathrm{~Hz}$, $1 \mathrm{H}), 7.41-7.31(\mathrm{~m}, 7 \mathrm{H}), 7.17(\mathrm{~d}, J=7.0 \mathrm{~Hz}, 1 \mathrm{H}), 7.13$ (d, $J=7.9 \mathrm{~Hz}, 1 \mathrm{H}), 6.93-6.90$ (m, 3 $\mathrm{H}), 6.84(\mathrm{t}, J=7.9 \mathrm{~Hz}, 1 \mathrm{H}), 6.19(\mathrm{~d}, J=7.9 \mathrm{~Hz}, 1 \mathrm{H}), 5.10\left(\mathrm{AB}, J=12.3 \mathrm{~Hz}, v_{\mathrm{ab}}=16.6 \mathrm{~Hz}, 2\right.$ H), $4.54(\mathrm{t}, J=7.9 \mathrm{~Hz}, 1 \mathrm{H}), 4.29$ (ddd, $J=11.8,8.3,2.6 \mathrm{~Hz}, 1 \mathrm{H}), 3.32(\mathrm{t}, J=12.2 \mathrm{~Hz}, 1 \mathrm{H})$, $2.80(\mathrm{~d}, J=12.7 \mathrm{~Hz}, 1 \mathrm{H}), 2.00(\mathrm{~m}, 1 \mathrm{H}), 1.41(\mathrm{~s}, 9 \mathrm{H}), 1.01(\mathrm{~d}, J=6.2 \mathrm{~Hz}, 3 \mathrm{H}), 0.91(\mathrm{~d}, J=6.2$ $\mathrm{Hz}, 3 \mathrm{H}) ;{ }^{13} \mathrm{C} \mathrm{NMR}\left(150 \mathrm{MHz}, \mathrm{CD}_{3} \mathrm{CN}\right) \delta 174.3,173.6,164.4,156.3,154.4,152.0,151.3,147.1$, $143.0,140.6,138.0,135.9,134.7,134.6,131.9,130.8,130.6,129.9,129.6,129.4,128.8,128.6$, $127.7,127.3,127.2,126.9,124.6,124.1,123.9,123.5,123.0,122.7,112.5,98.2,84.1,67.1,57.5$, 57.0, 56.4, 37.9, 30.9, 27.5, 19.7, 19.1; HRMS (MALDI-FTMS) calcd for $\mathrm{C}_{48} \mathrm{H}_{40} \mathrm{Cl}_{2} \mathrm{~N}_{6} \mathrm{O}_{9} \mathrm{Na}^{+}$[M $\left.+\mathrm{Na}^{+}\right]$937.2126, found 937.2136.

Phenolic lactam 50. Bis-chlorinated intermediate 49 (11.0 mg, $0.0117 \mathrm{mmol}, 1.0$ equiv) was treated with neat TFA $(1.0 \mathrm{~mL})$ at $25^{\circ} \mathrm{C}$. After stirring the resultant solution for 10 minutes at $25^{\circ} \mathrm{C}$, the solvent was removed in vacuo (using several benzene co-evaporations to ensure the complete removal of all TFA) to give phenolic lactam $\mathbf{5 0}(8.6 \mathrm{mg}, 90 \%$ yield $)$ as an amorphous white solid. 50: $\mathrm{R}_{f}=0.35$ (silica gel, EtOAc/hexanes, 5:1); $[\alpha]_{\mathrm{D}}{ }^{20}=-277.3(c=0.40, \mathrm{MeOH})$; 
IR (film) $v_{\max } 3260,2919,1713,1660,1602,1496,1443,1401,1302,1261,1214,1155,1055$, $750 \mathrm{~cm}^{-1} ;{ }^{1} \mathrm{H} \mathrm{NMR}\left(600 \mathrm{MHz}, \mathrm{CD}_{3} \mathrm{CN}\right) \delta 10.38(\mathrm{~s}, 1 \mathrm{H}), 8.42(\mathrm{~s}, 1 \mathrm{H}), 7.54(\mathrm{~d}, J=7.9 \mathrm{~Hz}, 1 \mathrm{H})$, 7.42-7.33 (m, 7 H), 7.27 (br m, 1 H), 7.22 (d, J=7.0 Hz, 1 H), 7.10 (d, J=7.4 Hz, 1 H), 6.89$6.87(\mathrm{~m}, 2 \mathrm{H}), 6.82(\mathrm{t}, J=7.9 \mathrm{~Hz}, 1 \mathrm{H}), 6.77(\mathrm{~d}, J=7.9 \mathrm{~Hz}, 1 \mathrm{H}), 6.73(\mathrm{~s}, 1 \mathrm{H}), 6.15(\mathrm{~d}, J=7.9$ $\mathrm{Hz}, 1 \mathrm{H}), 5.08\left(\mathrm{AB}, J=12.7 \mathrm{~Hz}, \mathrm{v}_{\mathrm{ab}}=17.1 \mathrm{~Hz}, 2 \mathrm{H}\right), 4.55(\mathrm{t}, J=7.4 \mathrm{~Hz}, 1 \mathrm{H}), 4.24$ (ddd, $J=$ 11.4, 8.8, 3.1 Hz, $1 \mathrm{H}), 3.23(\mathrm{t}, J=12.7 \mathrm{~Hz}, 1 \mathrm{H}), 2.68$ (d, $J=11.4 \mathrm{~Hz}, 1 \mathrm{H}), 2.00$ (m, $1 \mathrm{H}), 1.01$ $(\mathrm{d}, J=6.1 \mathrm{~Hz}, 3 \mathrm{H}), 0.91(\mathrm{~d}, J=6.1 \mathrm{~Hz}, 3 \mathrm{H}) ;{ }^{13} \mathrm{C} \mathrm{NMR}\left(150 \mathrm{MHz}, \mathrm{CD}_{3} \mathrm{CN}\right) \delta 174.9,173.8$, $164.3,156.3,154.6,152.9,152.8,143.2,140.6,138.0,135.9,134.5,131.6,130.6,130.0,129.4$, $129.3,128.8,128.6,128.2,127.2,126.9,124.8,124.5,123.2,122.7,116.6,112.4,98.3,67.0$, 57.8, 57.0, 56.4, 37.8, 31.0, 19.6, 19.0; HRMS (MALDI-FTMS) calcd for $\mathrm{C}_{43} \mathrm{H}_{32} \mathrm{Cl}_{2} \mathrm{~N}_{6} \mathrm{O}_{7} \mathrm{H}^{+}$[M $\left.+\mathrm{H}^{+}\right]$815.1782, found 815.1778.

Aminal 52. A portion of phenolic lactam $50(5.0 \mathrm{mg}, 0.0061 \mathrm{mmol}, 1.0$ equiv) was dissolved in THF $(5 \mathrm{~mL})$ and treated with DIBAL-H $(0.012 \mathrm{~mL}, 1.0 \mathrm{M}$ in toluene, $0.012 \mathrm{mmol}$, 2.0 equiv) at $-78^{\circ} \mathrm{C}$. The reaction flask contents were then warmed to $25{ }^{\circ} \mathrm{C}$ over the course of 30 minutes. Upon completion, the reaction mixture was cooled back down to $-78{ }^{\circ} \mathrm{C}$, treated with another portion of DIBAL-H $(0.012 \mathrm{~mL}, 1.0 \mathrm{M}$ in toluene, $0.012 \mathrm{mmol}, 2.0$ equiv), and again allowed to warm to $25^{\circ} \mathrm{C}$ over the course of 30 minutes. This cycle was repeated three more times, and, once accomplished, the reaction mixture was quenched at $25{ }^{\circ} \mathrm{C}$ by the slow and careful addition of water $(5 \mathrm{~mL})$, poured into water $(25 \mathrm{~mL})$, and extracted with EtOAc $(3 \times$ $25 \mathrm{~mL})$. The combined organic layers were then washed with water $(25 \mathrm{~mL})$ and brine $(50 \mathrm{~mL})$, dried $\left(\mathrm{MgSO}_{4}\right)$, and concentrated. The resultant yellow residue was purified by preparative TLC (silica gel, EtOAc/hexanes, 1:1) to give aminal $\mathbf{5 2}\left(2.7 \mathrm{mg}, 56 \%\right.$ yield) as a clear film. 52: $\mathrm{R}_{f}=$ 0.40 (silica gel, EtOAc/hexanes, 1:1); IR (film) $v_{\max }$ 3366, 2923, 2849, 1701, 1660, 1490, 1449, $1396,1376,1343,1255,1202,1120,750,597 \mathrm{~cm}^{-1} ;{ }^{1} \mathrm{H}$ NMR (600 MHz, $\left.\mathrm{CD}_{3} \mathrm{CN}\right) \delta 10.41(\mathrm{~s}, 1$ H), $7.51(\mathrm{~d}, J=7.0 \mathrm{~Hz}, 1 \mathrm{H}), 7.40(\mathrm{~m}, 5 \mathrm{H}), 7.31(\mathrm{dd}, J=7.0,6.6 \mathrm{~Hz}, 1 \mathrm{H}), 7.30(\mathrm{~s}, 1 \mathrm{H}), 7.20$ (d, $J=7.0 \mathrm{~Hz}, 1 \mathrm{H}), 7.19(\mathrm{~d}, J=8.3 \mathrm{~Hz}, 1 \mathrm{H}), 6.98(\mathrm{~d}, J=6.6 \mathrm{~Hz}, 1 \mathrm{H}), 6.88(\mathrm{~d}, J=7.0 \mathrm{~Hz}, 1 \mathrm{H})$, $6.87(\mathrm{~d}, J=7.5 \mathrm{~Hz}, 1 \mathrm{H}), 6.78(\mathrm{~d}, J=7.8 \mathrm{~Hz}, 1 \mathrm{H}), 6.63(\mathrm{dd}, J=7.8,7.5 \mathrm{~Hz}, 1 \mathrm{H}), 6.38(\mathrm{~d}, J=$ 
$4.4 \mathrm{~Hz}, 1 \mathrm{H}), 6.17(\mathrm{~d}, J=8.0 \mathrm{~Hz}, 1 \mathrm{H}), 5.88(\mathrm{~d}, J=4.4 \mathrm{~Hz}, 1 \mathrm{H}), 5.09$ (s, $2 \mathrm{H}), 4.85$ (dd, $J=8.3$, $5.7 \mathrm{~Hz}, 1 \mathrm{H}), 4.17(\mathrm{~m}, 1 \mathrm{H}), 3.28(\mathrm{dd}, J=12.3,12.0 \mathrm{~Hz}, 1 \mathrm{H}), 2.80(\mathrm{dd}, J=12.3,3.0 \mathrm{~Hz}, 1 \mathrm{H})$, $2.11(\mathrm{~m}, 1 \mathrm{H}), 0.98$ (d, $J=7.2 \mathrm{~Hz}, 3 \mathrm{H}), 0.90$ (d, $J=7.2 \mathrm{~Hz}, 3 \mathrm{H})$; HRMS (MALDI-FTMS) calcd for $\mathrm{C}_{43} \mathrm{H}_{32} \mathrm{Cl}_{2} \mathrm{~N}_{6} \mathrm{O}_{6} \mathrm{Na}^{+}\left[\mathrm{M}+\mathrm{Na}^{+}\right]$821.1653, found 821.1669.

(-)-Diazonamide A (1). $\mathrm{Pd}(\mathrm{OH})_{2} / \mathrm{C}(0.002 \mathrm{~g}, 20 \mathrm{wt} \%$, catalytic $)$ was added to a solution of aminal $52\left(2.7 \mathrm{mg}, 0.0034 \mathrm{mmol}, 1.0\right.$ equiv) in $\mathrm{EtOH}(1 \mathrm{~mL})$ at $25^{\circ} \mathrm{C}$. The resultant suspension was then equipped with a balloon containing hydrogen gas (pressure of $\sim 2 \mathrm{~atm}$ ) and allowed to stir for 2 hours at $25^{\circ} \mathrm{C}$. Upon completion, the reaction flask contents were filtered through Celite and concentrated to give the desired amine $(2.1 \mathrm{mg}, 96 \%)$ as a clear oil. This intermediate $(2.1 \mathrm{mg}, 0.0032 \mathrm{mmol}, 1.0$ equiv) was then dissolved in DMF (1.0 mL) and treated sequentially at $25{ }^{\circ} \mathrm{C}$ with L-2-hydroxyisovaleric acid (1.9 mg, $0.0162 \mathrm{mmol}, 5.0$ equiv), $\mathrm{NaHCO}_{3}$ (4.0 mg, $0.0490 \mathrm{mmol}, 15$ equiv), $\mathrm{EDC} \cdot \mathrm{H}_{2} \mathrm{O}(3.0 \mathrm{mg}, 0.0162 \mathrm{mmol}, 5.0$ equiv), and $\mathrm{HOBt} \cdot \mathrm{H}_{2} \mathrm{O}\left(2.4 \mathrm{mg}, 0.0162 \mathrm{mmol}, 5.0\right.$ equiv). After 12 hours of stirring at $25^{\circ} \mathrm{C}$, the reaction flask contents were poured into saturated aqueous $\mathrm{NaHCO}_{3}(5 \mathrm{~mL})$ and extracted with EtOAc (3 $\times 5 \mathrm{~mL})$. The combined organic layers were then washed with $1 \mathrm{~N}$ aqueous $\mathrm{HCl}(5 \mathrm{~mL})$, water $(5$ $\mathrm{mL})$, and brine $(5 \mathrm{~mL})$, dried $\left(\mathrm{MgSO}_{4}\right)$, and concentrated. The resultant yellow residue was purified by preparative TLC (silica gel, EtOAc/hexanes, 1:1) to give (-)-diazonamide A (2.1 mg, $82 \%$ yield over two steps) as a clear film, a compound whose physical and spectral properties were identical in all respects with an authentic sample (based on ${ }^{1} \mathrm{H}$ NMR, optical rotation, HPLC, and TLC in a variety of solvents). ${ }^{1}$ For the purpose of direct comparison with C-37 epidiazonamide A (53), we are providing the ${ }^{1} \mathrm{H}$ NMR data that we obtained for synthetic $\mathbf{1}$ which is in complete accordance with that originally reported by Fenical, Clardy, and co-workers: ${ }^{1} \mathrm{H}$ NMR (600 MHz, CD 3 OD) $\delta 7.45(\mathrm{~d}, J=7.9 \mathrm{~Hz}, 1 \mathrm{H}), 7.36(\mathrm{dd}, J=8.3,7.3 \mathrm{~Hz}, 1 \mathrm{H}), 7.33(\mathrm{~s}, 1$ H), $7.19(\operatorname{app~d}, J=6.6 \mathrm{~Hz}, 2 \mathrm{H}), 7.00(\mathrm{~d}, J=7.4 \mathrm{~Hz}, 1 \mathrm{H}), 6.86(\mathrm{~d}, J=7.0 \mathrm{~Hz}, 1 \mathrm{H}), 6.77(\mathrm{~d}, J=$ $8.3 \mathrm{~Hz}, 1 \mathrm{H}), 6.66(\mathrm{dd}, J=7.4,7.3 \mathrm{~Hz}, 1 \mathrm{H}), 6.35(\mathrm{~s}, 1 \mathrm{H}), 4.85(\mathrm{~d}, J=6.7 \mathrm{~Hz}, 1 \mathrm{H}), 4.60(\mathrm{dd}, J=$ 11.5, $3.4 \mathrm{~Hz}, 1 \mathrm{H}), 3.88(\mathrm{dd}, J=3.9 \mathrm{~Hz}, 1 \mathrm{H}), 3.38(\mathrm{dd}, J=12.8,11.9 \mathrm{~Hz}, 1 \mathrm{H}), 2.78(\mathrm{dd}, J=$ 12.7, $3.5 \mathrm{~Hz}, 1 \mathrm{H}), 2.19$ (dqq, $J=6.4,6.9,6.9 \mathrm{~Hz}, 1 \mathrm{H}), 2.11(\mathrm{dqq}, J=3.3,6.8,6.7 \mathrm{~Hz}, 1 \mathrm{H})$, 
$1.05(\mathrm{~d}, J=6.6 \mathrm{~Hz}, 3 \mathrm{H}), 1.01(\mathrm{~d}, J=7.0 \mathrm{~Hz}, 3 \mathrm{H}), 0.96(\mathrm{~d}, J=6.5 \mathrm{~Hz}, 3 \mathrm{H}), 0.92(\mathrm{~d}, J=6.5 \mathrm{~Hz}$, $3 \mathrm{H})$.

C-37 epi-Diazonamide A (53). $\mathrm{Pd}(\mathrm{OH})_{2} / \mathrm{C}(0.002 \mathrm{~g}, 20 \mathrm{wt} \%$, catalytic $)$ was added to a solution of aminal $52\left(2.4 \mathrm{mg}, 0.0030 \mathrm{mmol}, 1.0\right.$ equiv) in $\mathrm{EtOH}(1 \mathrm{~mL})$ at $25^{\circ} \mathrm{C}$. The resultant suspension was then equipped with a balloon containing hydrogen gas (pressure of $\sim 2 \mathrm{~atm}$ ) and allowed to stir for 2 hours at $25^{\circ} \mathrm{C}$. Upon completion, the reaction flask contents were filtered through Celite and concentrated to give the desired amine (1.9 $\mathrm{mg}, 94 \%)$ as a clear oil. This intermediate (1.9 mg, $0.0029 \mathrm{mmol}, 1.0$ equiv) was then dissolved in DMF (1.0 mL) and treated sequentially at $25{ }^{\circ} \mathrm{C}$ with D-2-hydroxyisovaleric acid (1.7 mg, $0.0145 \mathrm{mmol}, 5.0$ equiv), $\mathrm{NaHCO}_{3}$ (3.6 mg, $0.0435 \mathrm{mmol}, 15$ equiv), $\mathrm{EDC} \cdot \mathrm{H}_{2} \mathrm{O}(2.7 \mathrm{mg}, 0.0145 \mathrm{mmol}, 5.0$ equiv), and $\mathrm{HOBt} \cdot \mathrm{H}_{2} \mathrm{O}\left(2.2 \mathrm{mg}, 0.0145 \mathrm{mmol}, 5.0\right.$ equiv). After 12 hours of stirring at $25{ }^{\circ} \mathrm{C}$, the reaction flask contents were poured into saturated aqueous $\mathrm{NaHCO}_{3}(5 \mathrm{~mL})$ and extracted with EtOAc (3 $\times 5 \mathrm{~mL})$. The combined organic layers were then washed with $1 \mathrm{~N}$ aqueous $\mathrm{HCl}(5 \mathrm{~mL})$, water $(5$ $\mathrm{mL})$, and brine $(5 \mathrm{~mL})$, dried $\left(\mathrm{MgSO}_{4}\right)$, and concentrated. The resultant yellow residue was purified by preparative TLC (silica gel, EtOAc/hexanes, 1:1) to give C-37 epi-diazonamide A (1.8 mg, 77\% yield over two steps) as a clear film. 53: ${ }^{1} \mathrm{H}$ NMR (600 MHz, $\left.\mathrm{CD}_{3} \mathrm{OD}\right) \delta 7.44(\mathrm{~d}, J$ $=7.8 \mathrm{~Hz}, 1 \mathrm{H}), 7.36(\mathrm{dd}, J=8.3,7.3 \mathrm{~Hz}, 1 \mathrm{H}), 7.33(\mathrm{~s}, 1 \mathrm{H}), 7.19(\operatorname{app} \mathrm{d}, J=6.5 \mathrm{~Hz}, 2 \mathrm{H}), 7.00$ $(\mathrm{d}, J=7.4 \mathrm{~Hz}, 1 \mathrm{H}), 6.86(\mathrm{~d}, J=6.9 \mathrm{~Hz}, 1 \mathrm{H}), 6.77(\mathrm{~d}, J=8.3 \mathrm{~Hz}, 1 \mathrm{H}), 6.66(\mathrm{dd}, J=7.3,7.2 \mathrm{~Hz}$, $1 \mathrm{H}), 6.34(\mathrm{~s}, 1 \mathrm{H}), 4.85(\mathrm{~d}, J=6.6 \mathrm{~Hz}, 1 \mathrm{H}), 4.62(\mathrm{dd}, J=11.5,3.4 \mathrm{~Hz}, 1 \mathrm{H}), 3.91(\mathrm{dd}, J=3.8$ $\mathrm{Hz}, 1 \mathrm{H}), 3.41$ (dd, $J=12.7,11.8 \mathrm{~Hz}, 1 \mathrm{H}), 2.77$ (dd, $J=12.7,3.5 \mathrm{~Hz}, 1 \mathrm{H}), 2.19$ (dqq, $J=6.4$, 6.9, $6.8 \mathrm{~Hz}, 1 \mathrm{H}), 2.11(\mathrm{dqq}, J=3.2,6.7,6.7 \mathrm{~Hz}, 1 \mathrm{H}), 1.04(\mathrm{~d}, J=6.7 \mathrm{~Hz}, 3 \mathrm{H}), 1.01(\mathrm{~d}, J=6.8$ $\mathrm{Hz}, 3 \mathrm{H}), 0.95(\mathrm{~d}, J=6.6 \mathrm{~Hz}, 3 \mathrm{H}), 0.91(\mathrm{~d}, J=6.7 \mathrm{~Hz}, 3 \mathrm{H}){ }^{2}$

\section{Notes}

1. An authentic sample was obtained from Professor Patrick G. Harran (who obtained it from Professor William Fenical) after the publication of our original communication regarding the first total synthesis of diazonamide A. 
Revised II

2. Copies of spectra are available upon request for all compounds presented in the supplemental material section of this manuscript. 